\title{
REMOVABLE SETS FOR HARMONIC
}

\author{
FUNCTIONS \\ IN BESOV SPACES
}

By DAVID KIGHURADZE

July, 2009 
REMOVABLE SETS FOR HARMONIC FUNCTIONS

IN BESOV SPACES

\author{
By \\ DAVID KIGHURADZE \\ Bachelor of Science \\ Tbilisi State University \\ Tbilisi,Georgia \\ 1984 \\ Master of Science \\ Tbilisi State University \\ Tbilisi, Georgia \\ 1988
}

Submitted to the Faculty of the

Graduate College of the

Oklahoma State University

in partial fulfillment of

the requirements for

the Degree of

DOCTOR OF PHILOSOPHY

July, 2009 


\section{REMOVABLE SETS FOR HARMONIC FUNCTIONS IN BESOV SPACES}

Thesis Approved:

Dr. David Ullrich

Thesis Advisor

Dr. Dale Alspach

Dr. Alan Noell

Dr. Igor Pritsker

Dr. Kaladi Babu

Dr. Gordon Emslie Dean of the Graduate College 


\section{ACKNOWLEDGMENTS}

First of all I would like to thank my advisor Dr. David Ullrich for his supervision and help with my studies. Dr. Ullrich has been a continual support throughout my research. Without his guidance I would not have been able to complete my dissertation and I am forever grateful to him. I truly hope that our collaboration will continue in future.

I would like to extend my appreciation to Dr. Dale Alspach who, as a graduate studies director, made it possible for me to come to Oklahoma State University and start my doctoral study. During all this time I always felt his friendly support and encouragement.

I am very grateful to my committee members for their assistance and encouragement during my studies. I would like to thank all the faculty members in the Mathematics Department at Oklahoma State University for the help and support I have received through these years.

I would like to express sincere gratitude to my fellow graduate students. They made my life enjoyable while studying here. Many of us developed close friendship which I hope continue after our graduations.

I would like to thank my parents and my sister for endless support and care. My special thanks go to my mother who was my math teacher during my early school years and who inspired me to be a mathematician. 
And finally I am deeply indebted to my wife for all her help, patience and ability to be always by my side. I am grateful to my children for making my life joyful in stressful and difficult times. Especially I would like to thank my oldest daughter who had warm and loving attitude towards me when I needed it the most. 


\section{TABLE OF CONTENTS}

1 Introduction 1

1.1 History of The Problem $\ldots \ldots \ldots \ldots \ldots$

1.2 Preliminaries . . . . . . . . . . . . . . . . . . 3

1.3 Basics About Besov Spaces . . . . . . . . . . . . . . . . . 5

2 Conditions of Non-Removability 23

2.1 Local Besov Spaces . . . . . . . . . . . . . . . . . . . . . . . . 23

2.2 Borderline Cases . . . . . . . . . . . . . . . . . . . . . . . . . 29

2.3 Constructing Functions in Besov Spaces Using Measures . . . . . . 33

$2.4 \quad \beta$ and Non-Removable Sets . . . . . . . . . . . . . . 36

3 Conditions of Removability $\quad 39$

3.1 Duality in Besov Spaces . . . . . . . . . . . . . . . . . 39

$3.2 \quad \beta$ and Removable Sets . . . . . . . . . . . . . . 44

3.3 Summary . . . . . . . . . . . . . . . . . 54

References ......................... 56 


\section{Introduction}

\subsection{History of The Problem}

Riemann's theorem about removable singularities states that if $f$ is holomorphic in $\Omega \backslash\{a\}$ ( $\Omega$ is an open set in $\mathbb{C}$ ) and $f$ is bounded in $D^{\prime}(a, r)$ (the punctured disk with radius $r$ and center $a$ ) for some $r>0$, then $f$ has a removable singularity at $a$. Riemann's theorem raises a question: How can we characterize removable singularities for "good" (analytic, harmonic, etc.) functions?

In 1945 L. Ahlfors [1] introduced the set function $\gamma$ ("analytic capacity"), and he proved that a compact set $K$ "is removable for bounded analytic functions", (that is for all open sets $U \supset K$ and all bounded analytic functions $f$ on $U \backslash K$ ) if and only if $\gamma(K)=0$.

The definition of $\gamma$ is purely analytic. So the question arises: What is a geometric characterization of sets with vanishing analytic capacity?

It is not difficult to show that if compact $K$ is thick enough, i.e. if its Hausdorff dimension $\operatorname{dim}(K)$ is strictly more than 1 then $\gamma(K)>0$. If $K$ is too small, i.e. $\operatorname{dim}(K)<1$ (then of course $m_{1}(K)=0$ ) or even $\operatorname{dim}(K)=1$ but $m_{1}(K)=0$ then $\gamma(K)=0\left(m_{\beta}\right.$ denotes the $\beta$ - dimensional Hausdorff measure of $\left.K\right)$.

In 1959 A. G. Vitushkin [17] gave an example of a compact set $K$ such that $m_{1}(K)>0$ but $\gamma(K)=0$ (Vitushkin's example was quite complicated but J. Garnett [6] and L. D. Ivanov [8] found a much simpler one). 
In 1967 Vitushkin conjectured that $\gamma(K)=0$ if and only if $m_{1}\left(\pi_{\theta}(K)\right)=0$ for almost every direction $\theta$, where $\pi_{\theta}$ denotes the orthogonal projection from the plane to the line $L_{\theta}=\{x+i y: x \cos (\theta)+y \sin (\theta)=0\}$.

Vitushkin's conjecture was confirmed [3] in case when $0<m_{1}(K)<\infty$.

In case when $m_{1}(K)=\infty$, P. Mattila [10], P. Jones and T. Murai [9] showed that Vitushkin's conjecture is not right.

M. Melnikov conjectured in 1995 that $\gamma(K)>0$ (this includes the case $\gamma=\infty$ ) if and only if $K$ supports a nontrivial positive measure with linear growth and finite Menger curvature [11].

Melnikov proved the "if" part of his own conjecture in the same article and the "only if" part was proved by X. Tolsa in 2003 [14].

As for harmonic functions, a theorem of Carleson [2] states that if $K$ is a compact subset of $\mathbb{R}^{d}(d \geq 2)$ then $K$ is removable for harmonic functions satisfying a $\operatorname{Lip}_{\alpha}$ condition $(0<\alpha<1)$ if and only if $m_{d-2+\alpha}(K)=0$.

Carleson's result fails for $\alpha=1$ : N. X. Uy [16] gave an example of a compact subset of $\mathbb{R}^{d}$ that is removable for $\operatorname{Lip}_{1}$ harmonic functions in spite of having positive $(d-1)$-dimensional Hausdorff measure. N. X. Uy noted that for $d=2$ the existence of such a compact set follows from the existence of a set of positive length that is removable for bounded holomorphic functions. Uy used the above-mentioned example of Garnett in the 2-dimensional case and he generalized this example in higher dimensional Euclidean spaces.

D. Ullrich showed in 1990 [15] that $K$ is removable for harmonic functions in the Zygmund class if and only if $m_{d-1}(K)=0$. 
When $0<\alpha<1$ then $\operatorname{Lip}_{\alpha}$ coincides with the Besov space $\Lambda_{\alpha}^{\infty, \infty}$ (see definition below) and the Zygmund class coincides with the Besov space $\Lambda_{1}^{\infty, \infty}$.

All these facts indicate that Carleson-Ullrich's theorem may have generalizations in more general Besov spaces. This dissertation consists of research done in this direction. We study removable sets for harmonic functions in Besov spaces.

The main result of this dissertation is:

Consider $\alpha, p, q$ such that $\left(2-\frac{d}{p^{\prime}}\right)_{+}<\alpha<2,1 \leq p \leq \infty, 1 \leq q \leq \infty$, and a compact $K \subset \mathbb{R}^{d}$. We fix the number $\beta=(\alpha-2) p^{\prime}+d$ where $\frac{1}{p^{\prime}}+\frac{1}{p}=1$. Then:

i) If there exists $\gamma<\beta$ such that $m_{\gamma}(K)=0$ then $K$ is $\Lambda_{\alpha, l o c}^{p, q}-$ removable;

ii) If there exists $\gamma>\beta$ such that $m_{\gamma}(K)>0$ then $K$ is not $\Lambda_{\alpha, l o c}^{p, q}-$ removable. (See the definitions below.)

\subsection{Preliminaries}

In this section we state basic definitions and theorems which are instrumental in our research. We start with notations: $\mathbb{R}^{d}$ denotes $d$-dimensional Euclidean space, $\mathbb{Z}$ the set of all integers, $\mathbb{N}$ the set of all natural numbers, $\mathbb{R}$ the set of all real numbers, $\mathbb{C}$ the set of complex numbers.

We denote by $A_{1}$ the "area" of the unit sphere in $\mathbb{R}^{d}$.

Definition 1.2.1. By g we always denote the Green function [7] which is defined in $\mathbb{R}^{d}$ as

$$
g(x)= \begin{cases}\frac{-1}{(d-2) A_{1}|x|^{d-2}}, & \text { if } x \neq 0, \\ -\infty, & \text { if } x=0,\end{cases}
$$


when $d>2$,

$$
g(x)= \begin{cases}\frac{-1}{2 \pi} \ln |x|, & \text { if } x \neq 0, \\ -\infty, & \text { if } x=0\end{cases}
$$

when $d=2$ and

$$
g(x)=\frac{1}{2}|x|
$$

when $d=1$.

Definition 1.2.2. The Fourier transform of $f \in L^{1}\left(\mathbb{R}^{d}\right)$ is

$$
\hat{f}(\xi)=\int_{\mathbb{R}^{d}} f(x) e^{-2 \pi i \xi \cdot x} d x
$$

For the further use, we need to make following

Remark 1.2.1. For all $d \in \mathbb{N}$, it is true that $\hat{g}(\xi)=-\frac{1}{4 \pi^{2}|\xi|^{2}}$ in $\mathbb{R}^{d} \backslash\{0\}$.

Theorem 1.2.1. Suppose that $\mu$ is a finite positive measure on $\mathbb{R}^{d}$ with compact support $K$. Let $u=\mu * g$, where $g$ is the Green's function (see definition 1.2.1). Then $u$ is subharmonic, and $\Delta u=\mu$ in the sense of distributions (so that $u$ is harmonic in $\left.\mathbb{R}^{d} \backslash K\right)[7]$

We denote by $m_{\theta}(E)$ the $\theta$-dimensional Hausdorff measure of $E \subset \mathbb{R}^{d}$. We state a special case of Frostman's lemma in Euclidean spaces:

Lemma 1.2.1. Suppose that $K$ is a compact subset of $\mathbb{R}^{d}$ and $m_{\theta}(K)>0$. Then there exist a probability measure $\mu$ supported on $K$ and a finite constant $C$ such that

$$
\mu(B(x, r)) \leq C r^{\theta}
$$


for all $x \in \mathbb{R}^{d}, r>0$.

Definition 1.2.3. Suppose $\mu$ is a measure in $\mathbb{R}^{d}$. We denote by $\mu_{r}$ the function $\mu_{r}(x)=\mu(B(x, r))$.

The next theorem is known as Hardy's inequality [4].

Theorem 1.2.2. If $\gamma>0,1 \leq q<\infty, h:(0, \infty) \rightarrow(0, \infty)$ then

$$
\int_{0}^{\infty} r^{-\gamma-1}\left(\int_{0}^{r} h(t) d t\right)^{q} d r \leq\left(\frac{q}{r}\right)^{q} \int_{0}^{\infty} r^{q-\gamma-1} h(r)^{q} d r
$$

\subsection{Basics About Besov Spaces}

$\mathcal{S}=\mathcal{S}\left(\mathbb{R}^{d}\right)$ will be the Schwarz space of rapidly decreasing functions, with dual $\mathcal{S}^{\prime}$, the space of tempered distributions. We will set

$$
\mathcal{S}_{0}=\left\{f \in \mathcal{S}: \int_{\mathbb{R}^{d}} f(t) t^{\gamma} d t=0, \gamma \in \mathbb{N}^{d}\right\}
$$

so

$$
\hat{\mathcal{S}}_{0}=\left\{\hat{f}: f \in \mathcal{S}_{o}\right\}=\left\{f \in \mathcal{S}: \frac{\partial^{\gamma} f}{\partial x^{\gamma}}(0)=0, \gamma \in \mathbb{N}^{d}\right\}
$$

The dual of $\mathcal{S}_{0}$ is

$$
\mathcal{S}_{0}^{\prime}=\mathcal{S}^{\prime} / \mathcal{S}_{0}^{\perp}=\mathcal{S}^{\prime} / \mathcal{P}
$$

where $\mathcal{P}$ is the space of all polynomials.

For each $n \in \mathbb{Z}$, the notation $\mathbb{A}_{n}$ will refer to the annulus

$$
\mathbb{A}_{n}=\left\{\xi \in \mathbb{R}^{d}: 2^{n-1}<|\xi|<2^{n+1}\right\}
$$


Choose $\psi_{0} \in C^{\infty}\left(\mathbb{R}^{d}\right)$ such that $\psi_{0}>0$ on $\mathbb{A}_{0}$ while $\psi_{0}=0$ on $\mathbb{R}^{d} \backslash \mathbb{A}_{0}$. For $n \in \mathbb{Z}$ define

$$
\psi_{n}(\xi)=\psi_{0}\left(2^{-n} \xi\right)
$$

(so that $\psi_{n}$ vanishes off $\mathbb{A}_{n}$ ), and now define $\Phi_{n} \in \mathcal{S}$ by

$$
\hat{\Phi}_{n}(\xi)=\frac{\psi_{n}(\xi)}{\sum_{j=-\infty}^{\infty} \psi_{j}(\xi)} .
$$

It follows that $\hat{\Phi}_{n}$ vanishes off $\mathbb{A}_{n},\left\|\Phi_{n}\right\|_{1}=\left\|\Phi_{0}\right\|_{1}, \Phi_{n}(x)=2^{n d} \Phi_{0}\left(2^{n} x\right)$,

$$
\int_{\mathbb{R}^{d}} x^{\gamma} \Phi_{n}(x) d x=\frac{\partial^{\gamma} \hat{\Phi}_{n}}{\partial x^{\gamma}}(0)=0, \gamma \in \mathbb{N}^{d}
$$

so $\Phi_{n} \in \mathcal{S}_{0}$, (hence $\Phi_{n} * f$ is defined for $\left.f \in \mathcal{S}_{0}^{\prime}\right)$ and $\sum_{n=-\infty}^{\infty} \hat{\Phi}_{n}(\xi)=1\left(\xi \in \mathbb{R}^{d} \backslash\{0\}\right)$. Note that $\sum_{n=-\infty}^{\infty} \hat{\Phi}_{n}(0)=0$.

Since $1-\sum_{n=0}^{\infty} \hat{\Phi}_{n}$ is smooth and has compact support we can choose $\Psi \in \mathcal{S}$ such that $\hat{\Psi}=1-\sum_{n=0}^{\infty} \hat{\Phi}_{n}$; note that $\hat{\Psi}$ vanishes off $B(0,1)$ (the ball with center at the origin and radius 1 ).

Now $\hat{\Psi}+\sum_{n=0}^{\infty} \hat{\Phi}_{n}=1$ in all of $\mathbb{R}^{d}$, and in fact it is easy to show that

$$
\phi=\Psi * \phi+\sum_{n=0}^{\infty} \Phi * \phi
$$

with convergence in $\mathcal{S}$, for all $\phi \in \mathcal{S}$. It follows that

$$
f=\Psi * f+\sum_{n=0}^{\infty} \Phi_{n} * f
$$


in $\mathcal{S}^{\prime}$ for all $f \in \mathcal{S}^{\prime}$ (Statements about convergence in $\mathcal{S}^{\prime}$ and $\mathcal{S}_{0}^{\prime}$ refer to the weak* topology).

Typically when we write $f=\sum_{n=-\infty}^{\infty} f_{n}$ below it will be understood that we mean convergence in $\mathcal{S}_{0}^{\prime}$ and that $\hat{f}_{n}$ vanishes off $\mathbb{A}_{n}$.

Similarly, the notation $f=F+\sum_{n=0}^{\infty} f_{n}$ will be taken to imply convergence in $\mathcal{S}^{\prime}$, that $\hat{f}_{n}$ vanishes off $\mathbb{A}_{n}$, and that $\hat{F}$ vanishes off $B(0,1)$.

Note that $f_{n}$ and $F$ will always be infinitely differentiable, since $\hat{f}_{n}$ and $\hat{F}$ are distributions with compact support.

Definition 1.3.1. Suppose that $-\infty<\alpha<\infty$ and $p \in[1, \infty], q \in[1, \infty)$. The homogeneous Besov space $\bigwedge_{\alpha}^{p, q}=\bigcap_{\alpha}^{p, q}\left(\mathbb{R}^{d}\right)$ is the space of tempered distributions $f \in \mathcal{S}_{0}^{\prime}$ such that $f=\sum_{n=-\infty}^{\infty} f_{n}$ where

$$
\operatorname{supp}\left(\hat{f}_{n}\right) \subset \mathbb{A}_{n}
$$

and

$$
\sum_{n=-\infty}^{\infty}\left(2^{n \alpha}\left\|f_{n}\right\|_{p}\right)^{q}<\infty
$$

If $q=\infty$ this condition becomes $2^{n \alpha}\left\|f_{n}\right\|_{p} \leq C$ for all $n \in \mathbb{Z}$.

Definition 1.3.2. Suppose that $-\infty<\alpha<\infty$ and $1 \leq p \leq \infty, 1 \leq q<\infty$. The inhomogeneous Besov space $\Lambda_{\alpha}^{p, q}=\Lambda_{\alpha}^{p, q}\left(\mathbb{R}^{d}\right)$ is the space of tempered distributions $f \in \mathcal{S}^{\prime}$ such that $f=F+\sum_{n=0}^{\infty} f_{n}$, where

$$
\operatorname{supp}(\hat{F}) \subset\left\{\xi \in \mathbb{R}^{d}:|\xi| \leq 1\right\}, \operatorname{supp}\left(\hat{f}_{n}\right) \subset \mathbb{A}_{n}
$$


and

$$
\|F\|_{p}+\left(\sum_{n=0}^{\infty}\left(2^{n \alpha}\left\|f_{n}\right\|_{p}\right)^{q}\right)^{\frac{1}{q}}<\infty .
$$

If $q=\infty$ this condition becomes $F \in L^{p}$ and $2^{n \alpha}\left\|f_{n}\right\|_{p} \leq C$ for all $n \in \mathbb{N}$.

We will let $\AA_{\alpha}=\AA_{\alpha}^{\infty, \infty}$ and $\Lambda_{\alpha}=\Lambda_{\alpha}^{\infty, \infty}$.

Norms in $\AA_{\alpha}^{p, q}$ and $\Lambda_{\alpha}^{p, q}$ are introduced by formulas:

$$
\|f\|_{\alpha, p, q}^{\circ}= \begin{cases}\left(\sum_{n=-\infty}^{\infty}\left(2^{n \alpha}\left\|\Phi_{n} * f\right\|_{p}\right)^{q}\right)^{\frac{1}{q}} & \text { if } 1 \leq q<\infty, \\ \sup _{n \in \mathbb{Z}} 2^{n \alpha}\left\|\Phi_{n} * f\right\|_{p}, & \text { if } q=\infty,\end{cases}
$$

and

$$
\|f\|_{\alpha, p, q}= \begin{cases}\|\Psi * f\|_{p}+\left(\sum_{n=0}^{\infty}\left(2^{n \alpha}\left\|\Phi_{n} * f\right\|_{p}\right)^{q}\right)^{\frac{1}{q}} & \text { if } 1 \leq q<\infty, \\ \|\Psi * f\|_{p}+\sup _{n \in \mathbb{N}} 2^{n \alpha}\left\|\Phi_{n} * f\right\|_{p} & \text { if } q=\infty,\end{cases}
$$

correspondingly.

With respect to those norms Besov spaces are Banach spaces [12].

Note: We will be considering only the case $\alpha>0$; in this case if $f$ is in $\Lambda_{\alpha}^{p, q}$ or $\bigwedge_{\alpha}^{p, q}$ it followes that $f$ is locally integrable (see Lemma 2.2.3 below).

Definition 1.3.3. Lipschitz space (homogeneous) Lip ${ }_{\alpha}^{\circ}(0<\alpha \leq 1)$ is the subspace of $C\left(\mathbb{R}^{d}\right) / \mathbb{C}$ with norm

$$
\|f\|_{\text {Lip }}=\sup _{\substack{x, y \in \mathbb{R} \\ x \neq y}} \frac{|f(x)-f(y)|}{|x-y|^{\alpha}} .
$$


Definition 1.3.4. Lipschitz space (inhomogeneous) Lip $(0<\alpha \leq 1)$ is the subspace of $C\left(\mathbb{R}^{d}\right)$ with norm

$$
\|f\|_{L_{L i p}}=\|f\|_{\infty}+\sup _{\substack{x, y \in \mathbb{R} \\ x \neq y}} \frac{|f(x)-f(y)|}{|x-y|^{\alpha}}
$$

Definition 1.3.5. We say that $f \in Z y g^{\circ}$ (homogeneous Zygmund class) if $f: \mathbb{R}^{d} \rightarrow$ $\mathbb{C}$ is a continuous function satisfying

$$
|f(x-h)-2 f(x)+f(x+h)| \leq C|h|
$$

for all $h \in \mathbb{R}^{d}$.

Definition 1.3.6. We say $f \in Z y g$ (the Zygmund class) if $f: \mathbb{R}^{d} \rightarrow \mathbb{C}$ is bounded, measurable and satisfies

$$
|f(x-h)-2 f(x)+f(x+h)| \leq C|h|
$$

for all $h \in \mathbb{R}^{d}$.

The next theorem, which belongs to the folklore [12], gives relationships among Besov spaces, Lipschitz spaces and the Zygmund class:

Theorem 1.3.1. Lip $_{\alpha}^{\circ}=\AA_{\alpha}$ and Lip $_{\alpha}=\Lambda_{\alpha}$ when $0<\alpha<1 ; Z y g^{\circ}=\AA_{1}$ and $Z y g=\Lambda_{1}$

In addition to this theorem we note well known facts: $\operatorname{Lip}_{1}^{\circ} \subset Z y g^{\circ}\left(\operatorname{Lip}_{1} \subset Z y g\right)$ but $\operatorname{Lip}_{1}^{\circ} \neq Z y g^{\circ}\left(\operatorname{Lip}_{1} \neq Z y g\right)$. 
Definition 1.3.7. The Poisson kernel in $\mathbb{R}^{d}$ is the function $\mathbf{P}_{y}$ defined by

$$
\mathbf{P}_{y}(x)=p_{d} \frac{y}{\left(y^{2}+|x|^{2}\right)^{\frac{d+1}{2}}} \quad\left(x \in \mathbb{R}^{d}, y>0\right),
$$

where $p_{d}$ is chosen so that $\int_{\mathbb{R}^{d}} \mathbf{P}_{y}(x) d x=1$.

The Poisson integral of $f$ is the function in $\mathbb{R}_{+}^{d+1}$ defined by

$$
\mathbf{P}[f](x, y)=f * \mathbf{P}_{y}(x)
$$

where $\mathbb{R}_{+}^{d+1}$ is the upper half space of $\mathbb{R}^{d+1}$ :

$$
\mathbb{R}_{+}^{d+1}=\left\{(x, y): x \in \mathbb{R}^{d}, y>0\right\}
$$

Definition 1.3.8. If $u(x, y)$ is a function in $\mathbb{R}_{+}^{d+1}$ and $y>0$ then for $1 \leq p<\infty$ we have

$$
\mathbf{m}_{y, p}(u)=\left(\int_{\mathbb{R}^{d}}|u(x, y)|^{p} d x\right)^{\frac{1}{p}}
$$

and

$$
\mathbf{m}_{y, \infty}(u)=\sup _{x \in \mathbb{R}}|u(x, y)|
$$

for $p=\infty$.

We define the finite difference $\Delta_{h} f$ by

$$
\Delta_{h} f(x)=f(x+h)-f(x),
$$


and we define higher-order difference operators by induction:

$$
\Delta_{h}^{1} f=\Delta_{h} f, \quad \Delta_{h}^{k+1} f=\Delta_{h}\left(\Delta_{h}^{k} f\right) .
$$

Theorem 1.3.2. [12] Suppose that $\alpha>0, p \in[1, \infty], q \in[1, \infty), k \in \mathbb{Z}$, and $k>\alpha$. Let $u=\mathbf{P}[f]$. The following are equivalent:

(i) $f \in \stackrel{\circ}{\Lambda}_{\alpha}^{p, q}$,

(ii) $\left(\int_{\mathbb{R}^{d}}\left(\frac{\left\|\Delta_{h}^{k} f\right\|_{p}}{|h|^{\alpha}}\right)^{q} \frac{d h}{|h|^{d}}\right)^{\frac{1}{q}}<\infty$,

(iii) $\left(\int_{0}^{\infty}\left(\frac{y^{k} \mathbf{m}_{y, p}\left(\frac{\partial^{k} u}{\partial y^{k}}\right)}{y^{\alpha}}\right)^{q} \frac{d y}{y}\right)^{\frac{1}{q}}<\infty$,

(iv) If $\beta$ is any multi-index with $|\beta|=k$ then $\left(\int_{0}^{\infty}\left(\frac{y^{k} \mathbf{m}_{y, p}\left(\mathbf{D}^{\beta} u\right)}{y^{\alpha}}\right)^{q} \frac{d y}{y}\right)^{\frac{1}{q}}<\infty$,

(v) $\left(\int_{0}^{\infty}\left(\frac{y^{k} \mathbf{m}_{y, p}\left(\frac{\partial^{k} u}{\partial x_{j}^{k}}\right)}{y^{\alpha}}\right)^{q} \frac{d y}{y}\right)^{\frac{1}{q}}<\infty$ for $j=1, \ldots, d$.

When $q=\infty$ then (ii),(iii),(iv), and (v) should be interpreted as:

$\left(i i^{\prime}\right) \sup _{h \neq 0} \frac{\left\|\Delta_{h}^{k} f\right\|_{p}}{|h|^{\alpha}}<\infty$

$\left(i i i^{\prime}\right) \sup _{y>0} \frac{y^{k} \mathbf{m}_{y, p}\left(\frac{\partial^{k} u}{\partial y^{k}}\right)}{y^{\alpha}}<\infty$

$\left(i v^{\prime}\right) \sup _{y>0} \frac{y^{k} \mathbf{m}_{y, p}\left(\mathbf{D}^{\beta} u\right)}{y^{\alpha}}<\infty$

$\left(v^{\prime}\right) \sup _{y>0} \frac{y^{k} \mathbf{m}_{y, p}\left(\frac{\partial^{k} u}{\partial x_{j}^{k}}\right)}{y^{\alpha}}<\infty$ for $j=1, \ldots, d$.

Theorem 1.3.3. [12] Suppose that $\alpha>0, p \in[1, \infty], q \in[1, \infty), k \in \mathbb{Z}$, and $k>\alpha$.

Let $u=\mathbf{P}[f]$. The following are equivalent:

(i) $f \in \Lambda_{\alpha}^{p, q}$,

(ii) $f \in L^{p}$ and $\left(\int_{|h| \leq 1}\left(\frac{\left\|\Delta_{h}^{k} f\right\|_{p}}{|h|^{\alpha}}\right)^{q} \frac{d h}{|h|^{d}}\right)^{\frac{1}{q}}<\infty$ 
(iii) $f \in L^{p}$ and $\left(\int_{0}^{1}\left(\frac{y^{k} \mathbf{m}_{y, p}\left(\frac{\partial^{k} u}{\partial y^{k}}\right)}{y^{\alpha}}\right)^{q} \frac{d y}{y}\right)^{\frac{1}{q}}<\infty$

(iv) $f \in L^{p}$ and if $\beta$ is any multi-index with $|\beta|=k$ then $\left(\int_{0}^{1}\left(\frac{y^{k} \mathbf{m}_{y, p}\left(\mathbf{D}^{\beta} u\right)}{y^{\alpha}}\right)^{q} \frac{d y}{y}\right)^{\frac{1}{q}}<$ $\infty$,

$(v) f \in L^{p}$ and $\left(\int_{0}^{1}\left(\frac{y^{k} \mathbf{m}_{y, p}\left(\frac{\partial^{k} u}{\partial x_{j}^{k}}\right)}{y^{\alpha}}\right)^{q} \frac{d y}{y}\right)^{\frac{1}{q}}<\infty$ for $j=1, \ldots, d$.

When $q=\infty$ then (ii),(iii),(iv), and (v) should be interpreted as:

$\left(i i^{\prime}\right) f \in L^{p}$ and $\sup _{h \neq 0} \frac{\left\|\Delta_{h}^{k} f\right\|_{p}}{|h|^{\alpha}}<\infty$,

$\left(i i i^{\prime}\right) f \in L^{p}$ and $\sup _{y>0} \frac{y^{k} \mathbf{m}_{y, p}\left(\frac{\partial^{k} u}{\partial y^{k}}\right)}{y^{\alpha}}<\infty$,

$\left(i v^{\prime}\right) f \in L^{p}$ and $\sup _{y>0} \frac{y^{k} \mathbf{m}_{y, p}\left(\mathbf{D}^{\beta} u\right)}{y^{\alpha}}<\infty$,

$\left(v^{\prime}\right) f \in L^{p}$ and $\sup _{y>0} \frac{y^{k} \mathbf{m}_{y, p}\left(\frac{\partial^{k} u}{\partial x_{j}^{k}}\right)}{y^{\alpha}}<\infty$ for $j=1, \ldots, d$.

The following theorem is known as the Besov Embedding Theorem [12]:

Theorem 1.3.4. If $1 \leq p \leq r \leq \infty$ and $\alpha=\beta+\frac{1}{p}-\frac{1}{r}$ then

$$
\Lambda_{\alpha}^{p, q} \subset \Lambda_{\beta}^{r, q}
$$

and

$$
\stackrel{\Lambda}{\Lambda}_{\alpha}^{p, q} \subset \stackrel{\curlywedge}{\Lambda}_{\beta}^{r, q}
$$

for all $q \in[1, \infty]$.

The next two lemmas will be very useful for us in the future [12].

Lemma 1.3.1. $f \in \AA_{\alpha}^{p, q}$ if and only if $D^{\gamma}(f) \in \stackrel{\Lambda}{\alpha-|\gamma|}_{\alpha, q}$ where $-\infty<\alpha<\infty, 1 \leq p \leq$ $\infty, 1 \leq q \leq \infty$ and $\gamma \in \mathbb{N}^{d}$ 
Lemma 1.3.2. If $f \in \Lambda_{\alpha}^{p, q}$ then $D^{\gamma}(f) \in \Lambda_{\alpha-|\gamma|}^{p, q}$ where $-\infty<\alpha<\infty, 1 \leq p \leq$ $\infty, 1 \leq q \leq \infty$ and $\gamma \in \mathbb{N}^{d}$

Before we state the theorem about duals of the Besov spaces [12], we need to introduce the next notations.

Suppose $h \in \Lambda_{-\alpha}^{p^{\prime}, q^{\prime}}$ and $f \in \Lambda_{\alpha}^{p, q}$ where as always below $p^{\prime}$ and $q^{\prime}$ are the conjugate exponents to $p$ and $q$, respectively. Note that

$$
\int_{\mathbb{R}^{d}}\left(\Phi_{n} * f\right)(x)\left(\Phi_{m} * h\right)(x) d x=0
$$

when $|n-m|>1$, because the (distribution) Fourier transforms have disjoint support. Similarly

$$
\int_{\mathbb{R}^{d}}(\Psi * f)(x)\left(\Phi_{n} * h\right)(x) d x=\int_{\mathbb{R}^{d}}\left(\Phi_{n} * f\right)(x)(\Psi * h)(x) d x=0
$$

for $n \geq 1$. We define a linear functional $\lambda_{h}: \Lambda_{\alpha}^{p, q} \rightarrow \mathbb{C}$ by a formula

$$
\begin{aligned}
\lambda_{h}(f) & =\int_{\mathbb{R}^{d}}(\Psi * f)(x)(\Psi * h)(x) d x+\int_{\mathbb{R}^{d}}\left(\Phi_{0} * f\right)(x)(\Psi * h)(x) d x+\int_{\mathbb{R}^{d}}(\Psi * f)(x)\left(\Phi_{0} * h\right)(x) d x \\
& +\int_{\mathbb{R}^{d}}\left(\Phi_{0} * f\right)(x)\left(\Phi_{0} * h\right)(x) d x+\sum_{j=-1} \sum_{n=1}^{\infty} \int_{\mathbb{R}^{d}}\left(\Phi_{n} * f\right)(x)\left(\Phi_{n+j} * h\right)(x) d x .
\end{aligned}
$$

The norm closure of the Schwarz space in $\Lambda_{\alpha}^{p, q}$ we denote by $\Lambda_{\alpha, 0}^{p, q}$ and observe that $\Lambda_{\alpha}^{p, q}=\Lambda_{\alpha, 0}^{p, q}$ except when $q=\infty$

Theorem 1.3.5. Suppose $\alpha \in \mathbb{R}, 1 \leq q \leq \infty, 1 \leq p \leq \infty$. If $\lambda \in\left(\Lambda_{\alpha, 0}^{p, q}\right)^{*}$ then there exists $h \in \Lambda_{-\alpha}^{p^{\prime}, q^{\prime}}$ with $\lambda=\lambda_{h}$. 
An atomic decomposition of distributions from Besov spaces, introduced by $M$. Frazier and B. Jawerth [5], plays a very important role in our research.

Definition 1.3.9. An $(\alpha, p)$ - atom $a(x),(-\infty<\alpha<\infty, 0<p \leq \infty)$ is a function satisfying, for some cube $Q \subseteq \mathbb{R}^{d}$, the following conditions:

(i) $\operatorname{supp}(a) \subseteq 3 Q$

(ii) $\left|D^{\gamma} a(x)\right| \leq|Q|^{\frac{\alpha}{d}-\frac{1}{p}-\frac{|\gamma|}{d}}$ if $|\gamma| \leq K$, and $x \in \mathbb{R}^{d}$

(iii) $\int_{\mathbb{R}^{d}} x^{\gamma} a(x) d x=0$ if $|\gamma| \leq N$,

where $K \geq([\alpha]+1)_{+}$and $N \geq \max \left(\left[d\left(\frac{1}{p}-1\right)_{+}-\alpha\right],-1\right)$ are fixed integers.

Here $x_{+}=\max (x, 0),[x]$ is the greatest integer in $x$, and $3 Q$ is the cube in $\mathbb{R}^{d}$ concentric with $Q$ but with side length three times of $Q$. If $N=-1$ then $a(x)$ is not required to have any vanishing moments.

We note that if $\alpha>0$ and $p \geq 1$ then we may take $N=-1$ so that condition (iii) may be ignored.

We write $a_{Q}$ for an atom satisfying the definition 1.3.9 for a given cube $Q$, and adopt the convention that whenever $Q$ appears as a summation index, the sum runs only over dyadic cubes. We also need for future use the following notations: Fix $\psi \in \mathcal{S}$ satisfying $\operatorname{supp}(\hat{\psi}) \subseteq\left\{\xi \in \mathbb{R}^{d}:|\xi| \leq \pi\right\}, \quad \int_{\mathbb{R}^{d}} x^{\eta} \psi(x) d x=0$ if $|\eta| \leq N$, and $\hat{\psi}(\xi) \geq c>0$ if $1 / 2 \leq|\xi| \leq 2$ ( $N$ is the fixed integer above). For each $\nu \in \mathbb{Z}$ and $k=\left(k_{1}, \ldots, k_{d}\right) \in \mathbb{Z}^{d}$, set

$$
Q_{\nu k}=\left\{x=\left(x_{1}, \ldots, x_{d}\right) \in \mathbb{R}^{d}: k_{i} 2^{-\nu} \leq x_{i}<\left(k_{i}+1\right) 2^{-\nu}, i=1, \ldots, d\right\}
$$


and define

$$
\psi_{Q}(x)=|Q|^{\frac{\alpha}{d}-\frac{1}{p}} \psi\left(2^{\nu} x-k\right) \quad \text { if } Q=Q_{\nu k} .
$$

Definition 1.3.10. We call a function $m$ an $(\alpha, p)$-molecule if there exist $\mu \in \mathbb{Z}$ and a point $x_{0} \in \mathbb{R}^{d}$ such that

$$
\left|D^{\eta} m(x)\right| \leq 2^{\mu\left(\frac{d}{p}-\alpha+|\eta|\right)}\left(1+2^{\mu}\left|x-x_{0}\right|\right)^{-M-|\eta|} \text { if }|\eta| \leq K
$$

and

$$
\int_{\mathbb{R}^{d}} x^{\eta} m(x) d x=0 \text { if }|\eta| \leq N
$$

where $M \geq N+10 d$ and $K, N$ are as above.

Suppose $\Psi \in \mathcal{S}$ satisfies: $\operatorname{supp}(\hat{\Psi}) \subseteq\{\xi:|\xi| \leq \pi\}$ and $\hat{\Psi}(\xi) \geq C>0$ if $|\xi| \leq 1$.

Then the following decomposition result is true [5].

Theorem 1.3.6. Let $-\infty<\alpha<\infty, 0<p, q \leq \infty$.

a) Each $f \in \Lambda_{\alpha}^{p, q}$ can be decomposed as follows:

$$
\text { i) } f(\cdot)=\sum_{k \in \mathbb{Z}^{d}} s_{k} \Psi(\cdot-k)+\sum_{\nu=0}^{\infty} \sum_{l(Q)=2^{-\nu}} s_{Q} \psi_{Q}(\cdot) \text {, }
$$

where the $\psi_{Q}$ 's are defined by (1.2), or

$$
\text { ii) } f=\sum_{k \in \mathbb{Z}^{d}} s_{k} b_{k}+\sum_{\nu=0}^{\infty} \sum_{l(Q)=2^{-\nu}} s_{Q} a_{Q},
$$

where the $a_{Q}$ 's are $(\alpha, p)$-atoms, and the $b_{k}$ 's satisfy supp $b_{k} \subseteq 3 Q_{0 k}$ and $\left|D^{\eta} b_{k}(x)\right| \leq 1$ if $|\eta| \leq K$. In both cases the convergence is in $\mathcal{S}^{\prime}$, and

$$
\left(\sum_{k \in \mathbb{Z}^{d}}\left|s_{k}\right|^{p}\right)^{\frac{1}{p}}+\left(\sum_{\nu=0}\left(\sum_{l(Q)=2^{-\nu}}\left|s_{Q}\right|^{p}\right)^{\frac{q}{p}}\right)^{\frac{1}{q}} \leq C\|f\|_{\alpha, p, q},
$$


with $C$ independent of $f$.

b) Conversely, suppose $f=\sum_{k \in \mathbb{Z}^{d}} s_{k} m_{k}+\sum_{\nu=0}^{\infty} \sum_{l(Q)=2^{-\nu}} s_{Q} m_{Q}$, where each $m_{Q}$ is an $(\alpha, p)$-molecule concentrated on $Q$, and each $m_{K}$ satisfies $\left|D^{\eta} m_{k}(x)\right| \leq$ $(1+|x-k|)^{-M-|\eta|}$ if $|\eta| \leq K$, for some sufficiently large $M$.

Then

$$
\|f\|_{\alpha, p, q} \leq C\left(\sum_{k \in \mathbb{Z}^{d}}\left|s_{k}\right|^{p}\right)^{\frac{1}{p}}+C\left(\sum_{\nu=0}\left(\sum_{l(Q)=2^{-\nu}}\left|s_{Q}\right|^{p}\right)^{\frac{q}{p}}\right)^{\frac{1}{q}} .
$$

The next theorems on Besov spaces are crucial for our purpose to characterize removable sets for harmonic functions in Besov spaces. Theorem 1.3.7 is an unpublished result of D. Ullrich and by courtesy of the author we are providing the proofs in this thesis.

Definition 1.3.11. Suppose $f$ is a locally integrable function. For $r>0$ we define:

$$
S_{r} f(x)=f(x)-\int_{S} f(x+r \xi) d \sigma(\xi)
$$

where $\sigma$ is the rotation-invariant probability measure on $S$, the sphere of radius 1 .

Lemma 1.3.3. Suppose that $k>\frac{d}{2}$ is an integer. There exists $C>0$ such that for any $f \in C_{c}^{k}\left(\mathbb{R}^{d}\right)$ we have

$$
\|\hat{f}\|_{1} \leq C\|f\|_{2}^{1-\frac{d}{2 k}}\left(\sum_{i=1}^{d}\left\|\frac{\partial^{k} f}{\partial x_{i}^{k}}\right\|_{2}\right)^{\frac{d}{2 k}}
$$


Proof. We observe that we are using Hölder's inequality and Plancherel's theorem.

$$
\begin{aligned}
\int_{\mathbb{R}^{d}}|\hat{f}(x)| d x & =\int_{B(0, A)}|\hat{f}(x)| d x+\int_{\mathbb{R}^{d} \backslash B(0, A)}|\hat{f}(x)| d x \\
& =\int_{B(0, A)}|\hat{f}(x)| d x+\int_{\mathbb{R}^{d} \backslash B(0, A)} \frac{\left(\sum_{i=1}^{d}\left|x_{i}\right|^{k}\right)|\hat{f}(x)|}{\sum_{i=i}^{d}\left|x_{i}\right|^{k}} d x \\
& \leq C_{1} A^{\frac{d}{2}}\|f\|_{2}+C_{2}\left(\sum_{i=1}^{d}\left\|\frac{\partial^{k} f}{\partial x_{i}^{k}}\right\|_{2}\right) A^{-k+\frac{d}{2}} .
\end{aligned}
$$

Let

$$
A=\left(\frac{C_{2}\left(\sum_{i=1}^{d}\left\|\frac{\partial^{k} f}{\partial x_{i}^{k}}\right\|_{2}\right)}{C_{1}\|f\|_{2}}\right)^{\frac{1}{k}}
$$

Theorem 1.3.7. Suppose that $0<\alpha<2$. Then $f \in \Lambda_{\alpha}^{p, q}$ if and only if

$$
\int_{0}^{\infty}\left(\frac{\left\|S_{r} f\right\|_{p}}{r^{\alpha}}\right)^{q} \frac{d r}{r}<\infty
$$

which for $q=\infty$ should be taken to mean that $\frac{\left\|S_{r} f\right\|_{p}}{r^{\alpha}}$ is bounded for $0<r<\infty$.

Proof. We consider only the case $1 \leq q<\infty$; the case $q=\infty$ is very similar.

Assume that $f \in \stackrel{\circ}{\alpha}_{\alpha}^{p, q}$. Note that

$$
\begin{aligned}
S_{r} f(x) & =f(x)-\int_{S} f(x+r \xi) d \sigma(\xi) \\
& =f(x)-\int_{S} \frac{f(x+r \xi)+f(x-r \xi)}{2} d \sigma(\xi)
\end{aligned}
$$




$$
\begin{aligned}
& =-\frac{1}{2} \int_{S}(f(x+r \xi)-2 f(x)+f(x-r \xi)) d \sigma(\xi) \\
& =-\frac{1}{2} \int_{S} \Delta_{r \xi}^{2} f(x) d \sigma(\xi) .
\end{aligned}
$$

Therefore

$$
\left\|S_{r} f\right\|_{p} \leq C \int_{S}\left\|\Delta_{r h}^{2} f\right\|_{p} d \sigma(h),
$$

so that Hölder's inequality gives

$$
\left\|S_{r} f\right\|_{p}^{q} \leq C \int_{S}\left\|\Delta_{r h}^{2} f\right\|_{p}^{q} d \sigma(h),
$$

and an integration in polar coordinates shows that:

$$
\begin{aligned}
\int_{0}^{\infty}\left(\frac{\left\|S_{r} f\right\|_{p}}{r^{\alpha}}\right)^{q} \frac{d r}{r} & \leq C \int_{0}^{\infty} \frac{\int_{S}\left\|\Delta_{r h}^{2}\right\|_{p}^{q} d \sigma(h)}{r^{\alpha q}} \frac{d r}{r} \\
& =C \int_{0}^{\infty} \int_{S}\left(\frac{\left\|\Delta_{r h}^{2} f\right\|_{p}}{|r h|^{\alpha}}\right)^{q} \frac{1}{|r h|^{d}} d \sigma(h) r^{d-1} d r \\
& =C \int_{\mathbb{R}^{d}}\left(\frac{\left\|\Delta_{h}^{2} f\right\|_{p}}{|h|^{\alpha}}\right)^{q} \frac{d h}{|h|^{d}}<\infty .
\end{aligned}
$$

To finish the proof of the first part we use theorem 1.3.2.

To prove the second part it is sufficient to show that there exists $\delta>0$ and $C>0$ such that for all integers $n$ we have

$$
\left\|\Phi_{n} * f\right\|_{p} \leq C\left\|S_{r} f\right\|_{p}, \quad\left(2^{-n} \delta \leq r \leq 2^{-n+1} \delta\right) .
$$


We will prove this in case $n=0$; the general case follows by dilation.

From the definition of $\|f\|_{p, q, \alpha}^{\circ}$ it follows that

$$
\|f\|_{p, q, \alpha}^{\circ} \leq C\left(\int_{0}^{\infty}\left(\frac{\left\|S_{r} f\right\|_{p}}{r^{\alpha}}\right)^{q} \frac{d r}{r}\right)^{\frac{1}{q}}
$$

which is enough for the proof.

We have:

$$
\widehat{S_{r} f}=\nu_{r} \hat{f}
$$

where

$$
\nu_{r}(\xi)=1-\int_{S} e^{i r h \cdot \xi} d \sigma(h)=1-J(r|\xi|)
$$

where in turn

$$
J(r)=\int_{S} e^{i r \zeta_{1}} d \sigma(\zeta)
$$

It is clear that $J$ is smooth, and well-known that $J(r) \rightarrow 0$ as $r \rightarrow \infty$. (We are assuming that $d>1$ here; in the case $d=1$ there is nothing to prove, because $\left.S_{r} f=-\frac{\Delta_{r}^{2} f}{2}\right)$. So there exists $\delta>0$ such that $|J(r)|<\frac{1}{2}\left(r>\frac{\delta}{2}\right)$.

This implies that $\left|\nu_{r}(\xi)\right| \geq \frac{1}{2}\left(\delta \leq r \leq 2 \delta, \xi \in \mathbb{A}_{0}\right)$.

Now let $N$ be an integer larger than $\frac{d}{2}$. Since $J$ is smooth all its partial derivatives are locally bounded; it follows from the quotient rule and the fact that $\left|\nu_{r}\right| \geq \frac{1}{2}$ that

$$
\left|\frac{\partial^{\beta}}{\partial \xi^{\beta}} \frac{\widehat{\Phi}_{0}(\xi)}{\nu_{r}(\xi)}\right| \leq C \quad\left(\delta \leq r \leq 2 \delta, \xi \in \mathbb{A}_{0}\right)
$$


whenever $\beta$ is a multi-index with $|\beta| \leq N$. Since all our functions are supported in $\mathbb{A}_{0}$ it follows from Lemma 1.3.3 that there exists $\phi_{r} \in L^{1}$ such that

$$
\hat{\phi}_{r}=\frac{\hat{\Phi}_{0}}{\nu_{r}}
$$

and

$$
\left\|\phi_{r}\right\|_{1} \leq C \quad(\delta \leq r \leq 2 \delta) .
$$

This shows that

$$
f * \Phi_{0}=\phi_{r} *\left(S_{r} f\right),
$$

so that

$$
\left\|\Phi_{0} * f\right\|_{p} \leq\left\|\phi_{r}\right\|_{1}\left\|S_{r} f\right\|_{p} \leq C\left\|S_{r} f\right\|_{p}(\delta \leq r \leq 2 \delta) .
$$

That is exactly the inequality we need for $n=0$.

Theorem 1.3.7 plus a little argument gives the corresponding result for $\Lambda_{p, q}^{\alpha}$ :

Theorem 1.3.8. Suppose that $0<\alpha<2$. Then $f \in \Lambda_{\alpha}^{p, q}$ if and only if $f \in L^{p}$ and

$$
\int_{0}^{1}\left(\frac{\left\|S_{r} f\right\|_{p}}{r^{\alpha}}\right)^{q} \frac{d r}{r}<\infty
$$

which for $q=\infty$ should be taken to mean that $\frac{\left\|S_{r} f\right\|_{p}}{r^{\alpha}}$ is bounded for $0<r \leq 1$. 
Lemma 1.3.4. If $d>2$ then

$$
S_{r}(g)(x)= \begin{cases}\frac{-1}{A_{1}(d-2)}\left(r^{2-d}-|x|^{2-d}\right), & \text { if }|x| \leq r \\ 0, & \text { otherwise }\end{cases}
$$

where $A_{1}$ is the "area" of the unit sphere in $\mathbb{R}^{d}$.

When $d=2$ we have

$$
S_{r}(g)(x)= \begin{cases}\frac{-1}{2 \pi}(\ln |r|-\ln |x|), & \text { if }|x| \leq r \\ 0, & \text { otherwise }\end{cases}
$$

and when $d=1$ we have

$$
S_{r}(g)(x)= \begin{cases}\frac{1}{2}(|x|-r), & \text { if }|x| \leq r \\ 0, & \text { otherwise }\end{cases}
$$

Proof. We just give a proof when $d>2 ; d=2$ and $d=1$ are similar. The fact that $S_{r}(g)(x)=0$ for $|x|>r$ is clear since $g$ is harmonic in $\mathbb{R}^{d} \backslash\{0\}$. Note that $S_{r}(g)=g-g * \sigma_{r}$ where $\sigma_{r}$ is the rotation-invariant probability measure on $r S$, the sphere of radius $r$. It is clear that $\sigma_{r} * g$ is radial since $\sigma_{r}$ and $g$ are both radial (rotation invariant). 
Therefore, in $B(0, r)$ the function $\sigma_{r} * g$ is radial and harmonic, so it must be constant since it satisfies the mean value property; for $|x|<r$ we have

$$
\sigma_{r} * g(x)=\sigma_{r} * g(0)=\int_{S} g(r \xi) d \sigma(\xi)=\frac{-r^{2-d}}{A_{1}(d-2)}
$$

Thus $S_{r}(g)(x)=g(x)-\sigma_{r} * g(x)=\frac{-1}{A_{1}(d-2)}\left(r^{2-d}-|x|^{2-d}\right)$.

Lemma 1.3.5. $S_{r}(g)(x)=\frac{1}{A_{1}} \int_{0}^{r} t^{1-d} \mathbf{1}_{B(0, t)}(x) d t$ where

$$
\mathbf{1}_{B(0, t)}(x)= \begin{cases}1, & \text { if }|x|<t \\ 0, & \text { otherwise }\end{cases}
$$

Proof. We consider the case when $d>2$. The cases $d=1$ and $d=2$ are very similar. If $|x|>r$ it is clear that $\int_{0}^{r} t^{1-d} \mathbf{1}_{B(0, t)}(x) d t=0$. If $|x|<r$ then $\frac{1}{A_{1}} \int_{0}^{r} t^{1-d} \mathbf{1}_{B(0, t)}(x) d t=$ $\frac{1}{A_{1}} \int_{|x|}^{r} t^{1-d} d t=\frac{-1}{A_{1}(d-2)}\left(r^{2-d}-|x|^{2-d}\right)$. 


\section{Conditions of Non-Removability}

\section{$2.1 \quad$ Local Besov Spaces}

Definition 2.1.1. We say that a distribution $f$ belongs to local Besov space and write $f \in \Lambda_{\alpha, l o c}^{p, q}$ if for all $\varphi \in C_{c}^{\infty}$ we have $\varphi f \in \Lambda_{\alpha}^{p, q}$.

The next proposition gives an equivalent definition of local Besov spaces:

Theorem 2.1.1. Suppose $0<\alpha<2$, and $1 \leq p \leq \infty$. Then $f \in \Lambda_{\alpha, l o c}^{p, \infty}$ if and only if $f \in L_{\text {loc }}^{p}\left(\mathbb{R}^{d}\right)$ and for any compact $K \subset \mathbb{R}^{d}$ there exists $C>0$ such that

$$
\left(\int_{K}\left|\Delta_{h}^{2} f(x)\right|^{p} d x\right)^{\frac{1}{p}} \leq C|h|^{\alpha} \quad(0<|h| \leq 1) .
$$

Proof. We adopt the common convention that the letter $C$ denotes a constant, the value of which may vary from line to line. The "only if" part is trivial so we focus on the "if" part. Assume $f \in L_{l o c}^{p}\left(\mathbb{R}^{d}\right)$ and for any compact $K \subset \mathbb{R}^{d}$ there exists $C>0$ such that

$$
\left(\int_{K}\left|\Delta_{h}^{2} f(x)\right|^{p} d x\right)^{\frac{1}{p}} \leq C|h|^{\alpha}(0<|h| \leq 1) .
$$

Suppose $\phi \in C_{c}^{\infty}\left(\mathbb{R}^{d}\right)$; let $K=\operatorname{supp}(\phi), K_{1}=\bigcup_{x \in K} \overline{B(x, 2)}$. We know that $\Delta_{h}(\phi f)=\left(\Delta_{h} \phi\right)\left(\tau_{h} f\right)+\phi \Delta_{h} f$.

Applying that formula twice shows that

$$
\Delta_{h}^{2}(\phi f)=\left(\Delta_{h}^{2} \phi\right)\left(\tau_{2 h} f\right)+2\left(\Delta_{h} \phi\right)\left(\tau_{h} \Delta_{h} f\right)+\phi \Delta_{h}^{2} f .
$$


Since $\phi$ has compact support and the function $f$ is locally integrable everywhere it would be enough to give an $L^{p}$-norm estimate of

$$
\left(\Delta_{h}^{2} \phi\right) f+2\left(\Delta_{h} \phi\right)\left(\Delta_{h} f\right)+\phi \Delta_{h}^{2} f
$$

in order to have an $L^{p}$-norm estimate of $\Delta_{h}^{2}(\phi f)$. By Theorem 1.3.3 $\left(i i^{\prime}\right)$, it is enough to show that $\left\|\Delta_{h}^{2}(\phi f)\right\|_{p} \leq C|h|^{\alpha}$.

$$
\text { If }|h|>1 \text { then }
$$

$$
\left\|\Delta_{h}^{2}(\phi f)\right\|_{p} \leq 4\|\phi f\|_{p}=C \leq C|h|^{\alpha} .
$$

So we only need to consider the case $0<|h| \leq 1$.

In this case we have

$$
\left\|\left(\Delta_{h}^{2} \phi\right) f\right\|_{p} \leq\left\|\Delta_{h}^{2} \phi\right\|_{\infty}\left(\int_{K_{1}}|f|^{p}\right)^{\frac{1}{p}} \leq C|h|^{2} \leq C|h|^{\alpha},
$$

since $\phi \in C_{c}^{\infty}\left(\mathbb{R}^{d}\right)$ and $0<\alpha<2$. Also, it is easy to see that

$$
\left\|\phi \Delta_{h}^{2} f\right\|_{p} \leq C\left(\int_{K}\left|\Delta_{h}^{2} f\right|^{p}\right)^{\frac{1}{p}} \leq C|h|^{\alpha} .
$$


Thus we have the $\left(\Delta_{h} \phi\right)\left(\Delta_{h} f\right)$ term left to evaluate. We observe that $\Delta_{h}^{2} f=\left(\tau_{2 h} f-\right.$ $\left.\tau_{h} f\right)-\left(\tau_{h} f-f\right)$ so

$$
\begin{aligned}
\Delta_{2 h} f & =\left(\tau_{2 h} f-\tau_{h} f\right)+\left(\tau_{h} f-f\right) \\
& =\Delta_{h}^{2} f+2\left(\tau_{h} f-f\right) \\
& =\Delta_{h}^{2} f+2 \Delta_{h} f,
\end{aligned}
$$

or $\Delta_{h} f-\frac{1}{2} \Delta_{2 h} f=-\frac{1}{2} \Delta_{h}^{2} f$. Now assume that $2^{-N} \leq|h|<2^{-N+1}$. Then

$$
\begin{aligned}
\left\|\Delta_{h} f\right\|_{L^{p}\left(K_{1}\right)} \leq & \left\|\Delta_{h} f-\frac{1}{2} \Delta_{2 h} f\right\|_{L^{p}\left(K_{1}\right)} \\
& +\left\|\frac{1}{2} \Delta_{2 h} f-\frac{1}{4} \Delta_{4 h} f\right\|_{L^{p}\left(K_{1}\right)} \\
& +\cdots \\
& +\left\|2^{-N+1} \Delta_{2^{N-1} h} f-2^{-N} \Delta_{2^{N} h} f\right\|_{L^{p}\left(K_{1}\right)} \\
& +2^{-N}\left\|\Delta_{2^{N} h} f\right\|_{L^{p}\left(K_{1}\right)} \\
= & \frac{1}{2}\left\|\Delta_{h}^{2} f\right\|_{L^{p}\left(K_{1}\right)}+\cdots+2^{-N}\left\|\Delta_{2^{N-1} h}^{2} f\right\|_{L^{p}\left(K_{1}\right)}+2^{-N}\left\|\Delta_{2^{N} h} f\right\|_{L^{p}\left(K_{1}\right)} .
\end{aligned}
$$

Since $2^{N}|h|<2$, we can easily estimate the last term:

$$
2^{-N}\left\|\Delta_{2^{N} h} f\right\|_{L^{p}\left(K_{1}\right)} \leq 2|h|\|f\|_{L^{p}\left(K_{2}\right)}=C|h|
$$

where $K_{2}=\bigcup_{x \in K_{1}} \overline{B(x, 1)}$. 
Now we estimate the other terms:

$$
\begin{aligned}
& \frac{1}{2}\left\|\Delta_{h}^{2} f\right\|_{L^{p}\left(K_{1}\right)}+\cdots+2^{-N}\left\|\Delta_{2^{N-1} h}^{2} f\right\|_{L^{p}\left(K_{1}\right)} \\
& \leq C\left(\frac{1}{2}|h|^{\alpha}+\frac{1}{4}|2 h|^{\alpha}+\cdots+2^{-N}\left|2^{N-1} h\right|^{\alpha}\right) \\
& =C|h|^{\alpha}\left(1+2^{\alpha-1}+2^{2(\alpha-1)}+\cdots+2^{(N-1)(\alpha-1)}\right) \\
& \leq \begin{cases}C|h|^{\alpha}, & \text { if } 0<\alpha<1, \\
C N|h|^{\alpha} \leq C|h| \log \left(\frac{1}{|h|}\right), & \text { if } \alpha=1, \\
C|h|^{\alpha} 2^{N(\alpha-1)} \leq C|h| & \text { if } \alpha>1 .\end{cases}
\end{aligned}
$$

Adding in the last term shows that

$$
\left\|\Delta_{h} f\right\|_{L^{p}\left(K_{1}\right)}= \begin{cases}C|h|^{\alpha}+|h|, & \text { if } 0<\alpha<1, \\ C N|h|^{\alpha} \leq C|h|\left(1+\log \left(\frac{1}{|h|}\right)\right), & \text { if } \alpha=1, \\ C|h|^{\alpha} 2^{N(\alpha-1)} \leq C|h| & \text { if } \alpha>1 .\end{cases}
$$

Since $\operatorname{supp}\left(\Delta_{h} \phi\right) \subset K_{1},\left\|\Delta_{h} \phi\right\|_{\infty} \leq C|h|, 0<\alpha<2$, and $|h| \leq 1$, it follows that

$$
\begin{aligned}
& \left\|\left(\Delta_{h} \phi\right)\left(\Delta_{h} f\right)\right\|_{p} \leq C|h|\left\|\Delta_{h} f\right\|_{L^{p}\left(K_{1}\right)} \\
& \leq|h| \begin{cases}C|h|^{\alpha}+|h|, & \text { if } 0<\alpha<1, \\
C|h|\left(1+\log \left(\frac{1}{|h|}\right)\right), & \text { if } \alpha=1, \\
C|h| & \text { if } \alpha>1 .\end{cases} \\
& \leq C|h|^{\alpha} \text {. }
\end{aligned}
$$


Now we give a more general definition of local Besov spaces:

Definition 2.1.2. Suppose $\Omega$ is an open set in $\mathbb{R}^{d}$. We say that a distribution $f$ in $\Omega$ belongs to local Besov space with respect to $\Omega$ and write $f \in \Lambda_{\alpha, l o c}^{p, q}(\Omega)$ if for all $\varphi \in C_{c}^{\infty}(\Omega)$ we have $\varphi f \in \Lambda_{\alpha}^{p, q}$.

It is easy to see that an analog of Theorem 2.1.1 is true:

Theorem 2.1.2. Suppose $0<\alpha<2$, and $1 \leq p \leq \infty$. Then $f \in \Lambda_{\alpha, l o c}^{p, \infty}(\Omega)$ if and only if $f \in L_{l o c}^{p}(\Omega)$ and for any compact $K \subset \Omega$ there exists $C>0$ and $\delta>0$ such that

$$
\left(\int_{K}\left|\Delta_{h}^{2} f(x)\right|^{p} d x\right)^{\frac{1}{p}} \leq C|h|^{\alpha}, \quad(0<|h| \leq \delta) .
$$

We state some propositions which give relationships between local and global Besov spaces.

Proposition 2.1.1. For all $0<\alpha<2,1 \leq q \leq \infty$ and $1 \leq p \leq \infty$ we have

$$
\bigwedge_{\alpha}^{p, q} \subseteq \Lambda_{\alpha, l o c}^{p, q}
$$

Proof. Say $f \in \AA_{\alpha}^{p, q}, f=\sum_{n=-\infty}^{\infty} f_{n}$. Since $\left(\sum_{n=-\infty}^{-1} f_{n}\right)^{\wedge}$ has compact support, $\sum_{n=-\infty}^{-1} f_{n} \in$ $C^{\infty}\left(\mathbb{R}^{d}\right) \subset L_{l o c}^{p}$. Also $C^{\infty}\left(\mathbb{R}^{d}\right) \subset \Lambda_{\alpha, l o c}^{p, q}\left(\mathbb{R}^{d}\right)$ (this follows from the definition of local Besov spaces since $\left.C_{c}^{\infty}\left(\mathbb{R}^{d}\right) \subset \Lambda_{\alpha}^{p, q}\left(\mathbb{R}^{d}\right)\right)$. On the other hand because $\alpha>0$ we have $\sum_{n=1}^{\infty}\left\|f_{n}\right\|_{p}<\infty$. Since $\sum_{n=0}^{\infty} f_{n} \in \Lambda_{\alpha}^{p, q}$, it follows from Proposition 3.1.1 below that $\sum_{n=0}^{\infty} f_{n} \in \Lambda_{\alpha, l o c}^{p, q}$ 
Proposition 2.1.2. If $\alpha_{1}<\alpha_{2}$ than

$$
\Lambda_{\alpha_{2}, l o c}^{p, q}(\Omega) \subset \Lambda_{\alpha_{1}, l o c}^{p, q}(\Omega)
$$

for all $1 \leq q \leq \infty, 1 \leq p \leq \infty$ and any open set $\Omega \subset \mathbb{R}^{d}$.

Proof. The proof easily follows from the fact that $\Lambda_{\alpha_{2}}^{p, q} \subset \Lambda_{\alpha_{1}}^{p, q}$ when $\alpha_{1}<\alpha_{2}$ [11].

Proposition 2.1.3. Say $\Omega$ is an open subset of $\mathbb{R}^{d}$ and $K$ a compact subset of $\Omega$. Then $K$ is $\Lambda_{\alpha, l o c}^{p, q}(\Omega)$ removable if and only if it is $\Lambda_{\alpha, l o c}^{p, q}\left(\mathbb{R}^{d}\right)$-removable.

Proof. For the non-trivial direction, say $K$ is not $\Lambda_{\alpha, l o c}^{p, q}(\Omega)$-removable. Let $u \in$ $\Lambda_{\alpha, l o c}^{p, q}(\Omega), u$ harmonic in $\Omega \backslash K, u$ not harmonic in $\Omega$. Then $\Delta u \neq 0, \operatorname{supp}(\Delta u) \subseteq K$ (where $\Delta u$ is the distribution Laplacian of $u$ ). Since $\Delta u$ has compact support and the Green function $g$ belongs to $L_{l o c}^{1}$, there exists well defined $v=(\Delta u) * g$. Then $v$ is harmonic in $\mathbb{R}^{d} \backslash K$ but not in $\mathbb{R}^{d}$, because $\Delta v=\Delta u$. To finish the proof we only need to show $v \in \Lambda_{\alpha, l o c}^{p, q}\left(\mathbb{R}^{d}\right)$.

Fix $\varphi_{0} \in C_{c}^{\infty}(\Omega), \varphi_{0}=1$ on a neighborhood of $K$. Suppose $\varphi \in C_{c}^{\infty}\left(\mathbb{R}^{d}\right)$. Then $\varphi v=\varphi \varphi_{0} v+\varphi\left(1-\varphi_{0}\right) v$ but $\varphi\left(1-\varphi_{0}\right) v \in C_{c}^{\infty}\left(\mathbb{R}^{d}\right) \subset \Lambda_{\alpha}^{p, q}\left(\mathbb{R}^{d}\right)$ and $\Delta(u-v)=0$ in $\Omega$ so $u-v \in C^{\infty}(\Omega)$. Hence $\varphi \varphi_{0}(v-u) \in C_{c}^{\infty}\left(\mathbb{R}^{d}\right) \subset \Lambda_{\alpha}^{p, q}\left(\mathbb{R}^{d}\right)$. On the other hand, the fact that $u \in \Lambda_{\alpha, l o c}^{p, q}(\Omega)$ implies that $\varphi \varphi_{0} u \in \Lambda_{\alpha}^{p, q}\left(\mathbb{R}^{d}\right)$ since $\varphi \varphi_{0} \in C_{c}^{\infty}(\Omega)$. Thus

$$
\varphi v=\varphi \varphi_{0} v+\varphi\left(1-\varphi_{0}\right) v=\varphi \varphi_{0} u+\varphi \varphi_{0}(v-u)+\varphi\left(1-\varphi_{0}\right) v \in \Lambda_{\alpha}^{p, q}\left(\mathbb{R}^{d}\right)
$$

which implies that $v \in \Lambda_{\alpha, l o c}^{p, q}\left(\mathbb{R}^{d}\right)$. 
The previous propositions tell us that distributions from homogeneous and inhomogeneous Besov spaces are similar locally. Because of this, we will study removable compact sets for harmonic functions in local Besov spaces.

\subsection{Borderline Cases}

Before we move towards the general case $0<\alpha<2$, we are going to discuss borderline cases.

Lemma 2.2.1. If $\mu$ is a tempered distribution and $\hat{\mu}$ has compact support then $\mu \in$ $C^{\infty}\left(\mathbb{R}^{d}\right)[4]$

Corollary 2.2.1. If $\gamma>0$ then $\Lambda_{\gamma} \subseteq C\left(\mathbb{R}^{d}\right), \AA_{\gamma} \subseteq C\left(\mathbb{R}^{d}\right)$.

Proof. If $f \in \AA_{\gamma}$ then $f=\sum_{n=-\infty}^{\infty} f_{n}$, but the previous lemma implies that $\sum_{n=-\infty}^{-1} f_{n} \in$ $C^{\infty}\left(\mathbb{R}^{d}\right), f_{n} \in C^{\infty}\left(\mathbb{R}^{d}\right)(n \in \mathbb{N})$. On the other hand $\sum_{n=1}^{\infty}\left\|f_{n}\right\|_{\infty}<\infty$ since $\gamma>0$ which implies the second part of this corollary. The proof of the first part is very similar.

Corollary 2.2.2. If $\gamma>2$ then $\Lambda_{\gamma} \subseteq C^{2}\left(\mathbb{R}^{d}\right), \AA_{\alpha} \subseteq C^{2}\left(\mathbb{R}^{d}\right)$.

Proof. The proof easily follows from Lemma 1.3.1, Lemma 1.3.2 and the previous corollary.

Lemma 2.2.2. Suppose $\alpha>2+\frac{d}{p}$ and $1 \leq p \leq \infty$. Then $\stackrel{\Lambda}{\alpha}_{\alpha}^{p, \infty} \subseteq C^{2}\left(\mathbb{R}^{d}\right)$.

Proof. We know that $\AA_{\alpha}^{p, \infty} \subseteq \AA_{\alpha-\frac{d}{p}}$ by Theorem 1.3.4. The proof follows from the previous corollary.

Theorem 2.2.1. Suppose $\alpha>2+\frac{d}{p}$ and $1 \leq p \leq \infty$. Then $K$ is $\bigwedge_{\alpha}^{p, \infty}$-removable if and only if $K^{\circ}=\emptyset\left(K^{\circ}\right.$ is the interior of $\left.K\right)$. 
Proof. If $K^{\circ} \neq \emptyset$ then there exists $f \in C_{c}^{\infty}\left(\mathbb{R}^{d}\right)$ such that $f \neq 0$ and $\operatorname{supp}(f) \subseteq K$. To prove the second part we observe that since $\alpha>2+\frac{d}{p}$, any tempered distribution from $\AA_{\alpha}^{p, \infty}$ has continuous second order derivatives by the previous corollary and since interior of $K$ is empty it follows that $K$ is $\Lambda_{\alpha}^{p, \infty}$-removable.

An analogous result holds for non-homogeneous Besov spaces too. We can state in general:

Theorem 2.2.2. Suppose $\alpha>2+\frac{d}{p}$ and $1 \leq p \leq \infty$. Then $K$ is $\Lambda_{\alpha, l o c}^{p, \infty}-$ removable if and only if $K^{\circ}=\emptyset$.

Corollary 2.2.3. Suppose $\alpha>2+\frac{d}{p}, 1 \leq q \leq \infty$ and $1 \leq p \leq \infty$. Then $K$ is $\Lambda_{\alpha, l o c}^{p, q}-$ removable if and only if $K^{\circ}=\emptyset$.

Proof. The proof easily follows from the fact that $\Lambda_{\alpha, l o c}^{p, q} \subset \Lambda_{\alpha, l o c}^{p, \infty}$ when $\alpha>0$.

The next result considers the case when $\alpha>2$ but it gives only sufficient condition. We start with next

Lemma 2.2.3. Suppose $\alpha>0$ and $1 \leq p \leq \infty$. Then:

(i) $\Lambda_{\alpha}^{p, \infty} \subseteq L^{p}$

(ii) $\bigwedge_{\alpha}^{p, \infty} \subseteq L_{l o c}^{p}$

Proof. (i) Suppose $f \in \Lambda_{\alpha}^{p, \infty}$, and $f=F+\sum_{n=0}^{\infty} f_{n}$, then $F \in L^{p}$ and $\left\|f_{n}\right\|_{p} \leq C 2^{-n \alpha}$ which implies $\sum_{n=0}^{\infty}\left\|f_{n}\right\|_{p}<\infty$ since $0<\alpha$.

(ii) Suppose $f \in \bigwedge_{\alpha}^{p, \infty}$, and $f=\sum_{i=-\infty}^{\infty} f_{n}$. Then $\sum_{n=0}^{-\infty} f_{n} \in C^{\infty}$ and $\sum_{n=0}^{\infty} f_{n} \in L^{p}$, so $\bigwedge_{\alpha}^{p, \infty} \subseteq L_{l o c}^{p}$. 
Theorem 2.2.3. Suppose $\alpha>2$ and $1 \leq p \leq \infty$. If $m_{d}(K)=0$ then $K$ is $\Lambda_{\alpha, l o c}^{p, \infty}$ removable.

Proof. Say $f \in \Lambda_{\alpha, l o c}^{p, \infty}, f$ harmonic in $\mathbb{R}^{d} \backslash K, m_{d}(K)=0$. Then $\Delta f \in L_{l o c}^{p}, \Delta f=0$ a.e., so $\Delta f=0$.

Corollary 2.2.4. Suppose $\alpha>2,1 \leq q \leq \infty$ and $1 \leq p \leq \infty$. If $m_{d}(K)=0$ then $K$ is $\Lambda_{\alpha, l o c}^{p, q}$ removable.

Proof. The proof easily follows from the fact that $\Lambda_{\alpha, l o c}^{p, q} \subset \Lambda_{\alpha, l o c}^{p, \infty}$ when $\alpha>0$.

The converse of this theorem is true in the case when $\alpha=2$ but in the general case we do not know the answer.

Lemma 2.2.4. Suppose $1 \leq p \leq \infty$. If $f \in L^{p}$ then $f * g \in \stackrel{\complement}{\Lambda}_{2}^{p, \infty}$ where $g$ is the Green function (see definition 1.2.1).

Proof. We know that $\Delta(f * g)=f \in \AA^{p, \infty}$ so by Theorem 1.3 .2 we have $f * g \in$ $\bigwedge_{2}^{p, \infty}$

Theorem 2.2.4. Suppose $K$ is a compact subset of $\mathbb{R}^{d}$ and $1 \leq p \leq \infty$. If $m_{d}(K)>0$ then $K$ is not $\Lambda_{2, l o c}^{p, \infty}$-removable.

Proof. Since $m_{d}(K)>0$ there exists $f \in L^{p}$, such that $\int_{\mathbb{R}^{d}} f(x) d x=1$ and $\operatorname{supp}(f) \subseteq$ $K$. Then by Lemma 2.2.4 $f * g \in \Lambda_{2, l o c}^{p, \infty}$. So $K$ is not $\Lambda_{2, l o c}^{p, \infty}$-removable.

Now we turn our attention to the lower bound of the parameter $\alpha$.

Lemma 2.2.5. $g \in \bigwedge_{2-\frac{d}{p^{\prime}}}^{p, \infty}$ for any $p \in[1, \infty]$. 
Proof. We consider the case when $d>2$. The other cases are very similar to prove.

By Lemma 1.3.4

$$
S_{r}(g)(x)=\left\{\begin{array}{ll}
\frac{-1}{A_{1}(d-2)}\left(r^{2-d}-|x|^{2-d}\right), & \text { if }|x| \leq r, \\
0, & \text { otherwise }
\end{array} .\right.
$$

Since $2-\frac{d}{p^{\prime}}<2$ we may use Theorem 1.3.7 to finish the proof:

$$
\begin{aligned}
\frac{\left\|S_{r} g\right\|_{p}^{p}}{r^{\left(2-\frac{d}{p^{\prime}}\right) p}} & \leq C \frac{\int_{B(0, r)}|x|^{(2-d) p} d x}{r^{\left(2-\frac{d}{p^{\prime}}\right) p}} \\
& \leq C r^{(2-d) p+d-\left(2-\frac{d}{p^{\prime}}\right) p} \\
& =C
\end{aligned}
$$

Corollary 2.2.5. Suppose $\alpha=2-\frac{d}{p^{\prime}}$ and $p \in[1, \infty]$. Then $K$ is $\AA_{\alpha}^{p, \infty}$-removable if and only if $K=\emptyset$.

Recall that if $\gamma_{1}<\gamma_{2}$ then ${\stackrel{\Lambda}{\gamma_{2}, l o c}}^{p, \infty} \subset{\stackrel{\Lambda}{\gamma_{1}, l o c}}_{p, \infty}^{p}$ and $\AA_{\gamma}^{p, \infty} \subset \Lambda_{\gamma, l o c}^{p, \infty}$, which implies

Corollary 2.2.6. Suppose $\alpha \leq 2-\frac{d}{p^{\prime}}$ and $1 \leq p \leq \infty$. Then $K$ is $\Lambda_{\gamma, l o c}^{p, \infty}-$ removable if and only if $K=\emptyset$.

So the only interesting case is $\alpha>2-\frac{d}{p^{\prime}}$. 


\subsection{Constructing Functions in Besov Spaces Using Measures}

Proposition 2.3.1. Suppose that $\mu$ is a measure on $\mathbb{R}^{d}$ and there exists a constant $C>0$ such that $\frac{\left\|\mu_{r}\right\|_{p}}{r^{\alpha-2+d}}<C$ for $0<r \leq 1$ ( $\mu_{r}$ is given by definition 1.2.3). Then $\left\|S_{r}(u)\right\|_{p}<C r^{\alpha}$ for $0<r \leq 1$, where $u=\mu * g$ (g is the Green's function).

Proof. It is clear that

$S_{r}(u)=\mu * S_{r}(g)=\mu *\left(\frac{1}{A_{1}} \int_{0}^{r} t^{1-d} \mathbf{1}_{B(0, t)} d t\right)=\frac{1}{A_{1}} \int_{0}^{r} t^{1-d} \mu * \mathbf{1}_{B(0, t)} d t=\frac{1}{A_{1}} \int_{0}^{r} t^{1-d} \mu_{t} d t$

and so, by Minkowski's inequality,

$$
\begin{aligned}
\left\|S_{r}(u)\right\|_{p} & \leq \int_{0}^{r} t^{1-d}\left\|\mu_{t}\right\|_{p} d t \\
& \leq C \int_{0}^{r} t^{1-d} t^{d-2+\alpha} d t \\
& =C \int_{0}^{r} t^{-1+\alpha} d t \\
& =C r^{\alpha} .
\end{aligned}
$$

Proposition 2.3.2. Suppose $p \in[1, \infty], q \in[1, \infty), 0<\alpha<2$, and $\mu$ is a measure on $\mathbb{R}^{d}$. If $\int_{0}^{\infty}\left(\frac{\left\|\mu_{r}\right\|_{p}}{r^{\alpha+d-2}}\right)^{q} \frac{d r}{r}<\infty$ then $\mu * g \in \AA_{\alpha}^{p, q}$.

Proof. We know that $S_{r}(\mu * g)=\frac{1}{A_{1}} \int_{0}^{r} t^{1-d} \mu_{t} d t$. So $\left\|S_{r}(\mu * g)\right\|_{p} \leq C \int_{0}^{r} t^{1-d}\left\|\mu_{t}\right\|_{p} d t$. Suppose $\int_{0}^{\infty}\left(\frac{\left\|\mu_{r}\right\|}{r^{\alpha+d-2}}\right)^{q} \frac{d r}{r}<\infty$. Then Theorem 1.2.2 implies: 


$$
\begin{aligned}
\int_{0}^{\infty}\left(\frac{\left\|S_{r}(\mu * g)\right\|_{p}}{r^{\alpha}}\right)^{q} \frac{d r}{r} & \leq C \int_{0}^{\infty} r^{-\alpha q-1}\left(\int_{0}^{r} t^{1-d}\left\|\mu_{t}\right\|_{p} d t\right)^{q} d r \\
& \leq C \int_{0}^{\infty} r^{q-\alpha q-1}\left(r^{1-d}\left\|\mu_{r}\right\|_{p}\right)^{q} d r \\
& =C \int_{0}^{\infty}\left(\frac{\left\|\mu_{r}\right\|_{p}}{r^{\alpha+d-2}}\right)^{q} \frac{d r}{r} \\
& <\infty
\end{aligned}
$$

Thus by Theorem 1.3 .7 we have $\mu * g \in \AA_{\alpha}^{p, q}$.

Proposition 2.3.3. Suppose $\mu$ is a measure on $\mathbb{R}^{d}, 0<\alpha<2$. If $p \in[1, \infty], q \in$ $[1, \infty)$, and $\int_{0}^{1}\left(\frac{\left\|\mu_{r}\right\|_{p}}{r^{\alpha+d-2}}\right)^{q} \frac{d r}{r}<\infty$ then $\mu * g \in \Lambda_{\alpha}^{p, q}$.

The proof is exactly the same as of Proposition 2.3.2. To prove the converse of Propositions 2.3.1 and 2.3.2 we need next

Lemma 2.3.1. Suppose $\mu$ is a measure on $\mathbb{R}^{d}$ and $\alpha>0$. If $\left\|\mu_{r}\right\|_{p}>M r^{d+\alpha-2}$ for some $M>0$ and $r>0$ then there exists $C>0$ such that $\left\|S_{2 r}(g * \mu)\right\|_{p}>C M r^{\alpha}$.

Proof. We are considering the case $d>2$. Cases $d=1$ and $d=2$ are very similar.

We know that

$$
S_{r}(g)(x)= \begin{cases}\frac{-1}{(d-1) A_{1}}\left(r^{2-d}-|x|^{2-d}\right), & \text { if } x \leq r \\ 0, & \text { otherwise. }\end{cases}
$$


where $A_{1}$ is the area of the unit sphere. This implies that

$$
\left|S_{2 r}(g)(x)\right| \geq \frac{1}{(d-1) A_{1}}\left(\frac{1}{r^{d-2}}-\frac{1}{(2 r)^{d-2}}\right)=C \frac{1}{r^{d-2}}
$$

when $|x| \leq r$. Then

$$
\begin{aligned}
\left\|S_{2 r}(g * \mu)\right\|_{p} & =\left\|S_{2 r}(g) * \mu\right\|_{p} \\
& \geq \frac{C}{r^{d-2}}\left\|\mathbf{1}_{B(0, r)} * \mu\right\|_{p} \\
& =C r^{-d+2}\left\|\mu_{r}\right\|_{p} \\
& \geq C r^{-d+2+d-2+\alpha} M \\
& =C M r^{\alpha} .
\end{aligned}
$$

Corollary 2.3.1. Suppose $\mu$ is a measure in $\mathbb{R}^{d}$ supported on the compact set $K$ and $\alpha>0$. If for any $M>0$ there exists $r>0$ such that $\left\|\mu_{r}\right\|_{p} \geq M r^{d-2+\alpha}$ then for any $M_{1}>0$ there exists $r_{1}>0$ such that $\left\|S_{r_{1}}(\mu * g)\right\|_{p}>M_{1} r^{\alpha}$

Proof. This is clear from the previous lemma.

Corollary 2.3.2. Suppose $\mu$ is measure in $\mathbb{R}^{d}$ supported on the compact set $K$. If $\int_{0}^{1}\left(\frac{\left\|\mu_{r}\right\|_{p}}{r^{d-2+\alpha}}\right)^{q} \frac{d r}{r}=\infty$ then $\int_{0}^{1}\left(\frac{\left\|S_{r}(u)\right\|_{p}}{r^{\alpha}}\right)^{q} \frac{d r}{r}=\infty$ where $u=\mu * g$. 
Proof.

$$
\begin{aligned}
\int_{0}^{1}\left(\frac{\left\|S_{r}(u)\right\|_{p}}{r^{\alpha}}\right)^{q} \frac{d r}{r} & \geq C \int_{0}^{1}\left(\frac{\left\|\mu_{\frac{r}{2}}\right\|_{p} r^{-d+2}}{r^{\alpha}}\right)^{q} \frac{d r}{r} \\
& =C \int_{0}^{1}\left(\frac{\left\|\mu_{\frac{r}{2}}\right\|_{p}}{r^{\alpha-2+d}}\right)^{q} \frac{d r}{r} \\
& =C \int_{0}^{\frac{1}{2}}\left(\frac{\left\|\mu_{r}\right\|_{p}}{r^{\alpha-2+d}}\right)^{q} \frac{d r}{r} \\
& =\infty
\end{aligned}
$$

since $\int_{\frac{1}{2}}^{1}\left(\frac{\left\|\mu_{r}\right\|_{p}}{r^{\alpha-2+d}}\right)^{q} \frac{d r}{r}<\infty$.

\section{$2.4 \beta$ and Non-Removable Sets}

Theorem 2.4.1. Suppose $\mu$ is a probability measure supported on a compact set $K \subset \mathbb{R}^{d}$

(i) If $\frac{\left\|\mu_{r}\right\|_{p}}{r^{\alpha-2+d}} \leq C$ for some constant $C>0$ when $0<r \leq 1,1 \leq p \leq \infty$ and $0<\alpha<2$, then $\mu * g \in \Lambda_{\alpha}^{p, \infty}$ and $\mu * g$ has a non-removable singularity on $K$.

(ii) If $\int_{0}^{1}\left(\frac{\left\|\mu_{r}\right\|_{p}}{r^{\alpha-2+d}}\right)^{q} \frac{d r}{r}<\infty(0<\alpha, 1 \leq p \leq \infty, 1 \leq q<\infty)$ then $\mu * g \in \Lambda_{\alpha}^{p, q}$ and $\mu * g$ has a non-removable singularity on $K$.

Proof. (i) We have already shown in proving Proposition 2.3.1 that under given conditions we have $\left\|\mu * S_{r}(g)\right\|_{p} \leq C r^{\alpha}$. By Theorem 1.3.7 it follows that $\mu * g \in \Lambda_{\alpha}^{p, \infty}$.

On the other hand $|\mu * g(x)|=\left|\int_{\mathbb{R}^{d}} g(x-y) d \mu(y)\right| \leq C \frac{\mu(K)}{(|x|-\operatorname{diam}(K))^{d-2}}$ (when $|x|$ is big enough) which shows that $\mu * g(x) \rightarrow 0$ when $|x| \rightarrow \infty$. But $\mu * g$ is harmonic outside of $K$ and $\Delta(\mu * g)=\mu * \Delta g=\mu * \delta_{0}=\mu \neq 0$ (as a distribution). Therefore $\mu * g$ cannot be extended to a harmonic function in $\mathbb{R}^{d}$. 
(ii) We know from proposition 2.3.3 that $\mu * g \in \Lambda_{\alpha}^{p, q}$ and an argument similar to the final argument of part (i) finishes the proof.

Similarly we can prove the following

Theorem 2.4.2. Suppose $\mu$ is a probability measure supported on a compact set $K \subset \mathbb{R}^{d}$. If $\int_{0}^{\infty}\left(\frac{\left\|\mu_{r}\right\|_{p}}{r^{\alpha-2+d}}\right)^{q} \frac{d r}{r}<\infty(1 \leq q<\infty, 0<\alpha<2)$ then $\mu * g \in \stackrel{\complement}{\alpha}_{\alpha}^{p, q}$ and $\mu * g$ has a non-removable singularity on the $K$.

Now we can characterize non removable singularities for $\Lambda_{\alpha}^{p, \infty}$-spaces:

Theorem 2.4.3. Suppose $K$ is a compact subset of $\mathbb{R}^{d}, 0<\alpha<2$, and $1 \leq p \leq \infty$. If $m_{\beta}(K)>0$, where $\beta=(\alpha-2) p^{\prime}+d$, then $K$ is not $\Lambda_{\alpha}^{p, \infty}$ removable.

Proof. Lemma 1.2.1 implies that there exists a probability measure $\mu$ supported on the $K$ such that $\left\|\mu_{r}\right\|_{\infty} \leq C r^{\beta}$. Since $\left\|\mu_{r}\right\|_{1}=C r^{d} \mu(K)$, we have

$$
\left\|\mu_{r}\right\|_{p}^{p} \leq\left\|\mu_{r}\right\|_{\infty}^{p-1}\left\|\mu_{r}\right\|_{1} \leq C r^{(p-1) \beta+d}=C r^{p(\alpha+d-2)}
$$

or

$$
\left\|\mu_{r}\right\|_{p} \leq c r^{\alpha-2+d}
$$

Note that $(p-1) \beta+d=(p-1)\left((\alpha-2) p^{\prime}+d\right)+d=(p-1) p^{\prime}(\alpha-2)+d p=(\alpha-2+d) p$ since $(p-1) p^{\prime}=p$. Now we apply Theorem 2.4.1 which finishes the proof.

It follows that if $m_{\beta}(K)>0$ then $K$ is not $\Lambda_{\alpha, l o c}^{p, \infty}$ removable.

Corollary 2.4.1. Suppose $0<\alpha<2,1 \leq q \leq \infty$, and $1 \leq p \leq \infty$. If there exists $\gamma>\beta$ such that $m_{\gamma}(K)>0$ then $K$ is not $\Lambda_{\alpha, l o c}^{p, q}-$ removable. 
Proof. There exists $0<\alpha_{1}<2$ such that $\gamma=\left(\alpha_{1}-2\right) p^{\prime}+d$. Since $\beta<\gamma$ we have $\alpha<\alpha_{1}$ which implies that $\Lambda_{\alpha_{1}, l o c}^{p, \infty} \subset \Lambda_{\alpha, l o c}^{p, q}$. We apply the previous theorem to finish the proof. 


\section{Conditions of Removability}

\subsection{Duality in Besov Spaces}

Proposition 3.1.1. Suppose $\varphi \in \mathcal{S}\left(\mathbb{R}^{d}\right)$. Then for any $0<\alpha, 1 \leq p \leq \infty$, and $1 \leq q \leq \infty$ there exists $C=C(\alpha, \varphi)$ such that

$$
\|\varphi f\|_{p, q, \alpha} \leq C\|f\|_{p, q, \alpha}
$$

for all $f \in \Lambda_{\alpha}^{p, q}$.

Proof. Suppose $f$ is a distribution from $\Lambda_{\alpha}^{p, q}$. Then the first part of theorem 1.3.6 implies that $f$ can be presented as $f=\sum_{k \in \mathbb{Z}^{d}} s_{k} b_{k}+\sum_{\nu=0}^{\infty} \sum_{l(Q)=2^{-\nu}} s_{Q} a_{Q}$, where the $a_{Q}$ 's are $(\alpha, p)$-atoms, and the $b_{k}$ 's satisfy $\operatorname{supp}\left(b_{k}\right) \subseteq 3 Q_{0 k}$ and $\left|D^{\eta} b_{k}(x)\right| \leq 1$ if $|\eta| \leq K$. The convergence is in $\mathcal{S}^{\prime}$, and

$$
\left(\sum_{k \in \mathbb{Z}^{d}}\left|s_{k}\right|^{p}\right)^{\frac{1}{p}}+\left(\sum_{\nu=0}\left(\sum_{l(Q)=2^{-\nu}}\left|s_{Q}\right|^{p}\right)^{\frac{q}{p}}\right)^{\frac{1}{q}} \leq C\|f\|_{p, q, \alpha},
$$

with $C$ independent of $f$. Since $\varphi \in \mathcal{S}$ and $K$ is a fixed positive integer it is clear that there exists $A$ such that $\left\|D^{\eta}\left(\frac{\varphi b_{k}}{A}\right)\right\|_{\infty} \leq 1$ when $|\eta| \leq K$. Since $0<\alpha$ and $1 \leq p$ we do not worry about vanishing moments for $\frac{\varphi a_{Q}}{A}$ and so $\frac{\varphi a_{Q}}{A}$ is an atom. We write $\varphi f$ as $\varphi f=\sum_{k \in \mathbb{Z}^{d}} A s_{k} \frac{\varphi b_{k}}{A}+\sum_{\nu=0}^{\infty} \sum_{l(Q)=2^{-\nu}} A s_{Q} \frac{\varphi a_{Q}}{A}$. So we can use the second part of theorem 1.3.6 to conclude that $\|\varphi f\|_{p, q, \alpha} \leq C\|f\|_{p, q, \alpha}$. 
Lemma 3.1.1. Suppose $f$ is a tempered distribution. Then $f \in \Lambda_{\alpha, l o c}^{p, q}$ if and only if for any open bounded set $\Omega \in \mathbb{R}^{d}$ there exists $\tilde{f} \in \Lambda_{\alpha}^{p, q}$ such that $f=\tilde{f}$ in $\Omega$.

Proof. Suppose $f \in \Lambda_{\alpha, l o c}^{p, q}$ and $\Omega$ is an open, bounded set in $\mathbb{R}^{d}$. Consider a function $\psi \in C_{c}^{\infty}\left(\mathbb{R}^{d}\right)$ such that $\psi(x)=1$ when $x \in \Omega$. Then by Proposition 3.1.1 we can take $\tilde{f}=f \psi$.

To prove the second part, assume that $f$ is a tempered distribution and $\psi$ is an arbitrary function from $C_{c}^{\infty}\left(\mathbb{R}^{d}\right)$. Consider a bounded open set $\Omega \in \mathbb{R}^{d}$ such that $\operatorname{supp}(\psi) \in \Omega$. Then there exists $\tilde{f} \in \Lambda_{\alpha}^{p, q}$ with $f=\tilde{f}$ in $\Omega$. So by Proposition 3.1.1 $f \psi=\tilde{f} \psi \in \Lambda_{\alpha}^{p, q}$.

Lemma 3.1.2. There exists $C>0$ such that for any function $h \in L^{p}$ which satisfies the condition $\operatorname{supp}(\hat{h}) \subset \mathbb{A}_{n}$, we have $\|g * h\|_{p} \leq C 2^{-2 n}\|h\|_{p}$.

Proof. Let us consider a function $\varphi_{0} \in \mathcal{S}$ such that $\hat{\varphi}_{0} \in C_{c}^{\infty}$ and $\hat{\varphi}_{0}(\xi)=\frac{-1}{4 \pi^{2}|\xi|^{2}}$ when $\xi \in \mathbb{A}_{0}$. We define $\varphi_{n}(x)=2^{-2 n+n d} \varphi_{0}\left(2^{n} x\right)$. Then

$$
\hat{\varphi}_{n}(\xi)=2^{-2 n+n d-n d} \hat{\varphi}_{0}\left(2^{-n} \xi\right)=\frac{-2^{-2 n}}{4 \pi^{2} 2^{-2 n}|\xi|^{2}}=\frac{-1}{4 \pi^{2}|\xi|^{2}}, \quad\left(\xi \in \mathbb{A}_{n}\right)
$$

So $\hat{\varphi}_{n}$ coincides with $\hat{g}$ on the annulus $\mathbb{A}_{n}$ (see the remark on page 4 ). Thus

$$
\|g * h\|_{p}=\left\|\varphi_{n} * h\right\|_{p} \leq\left\|\varphi_{n}\right\|_{1}\|h\|_{p} \leq C 2^{-2 n}\left\|\varphi_{0}\right\|_{1}\|h\|_{p} \leq C 2^{-2 n}\|h\|_{p} .
$$

Lemma 3.1.3. Suppose $f \in \Lambda_{\alpha}^{p, q}$ where $-\infty<\alpha<\infty, 1 \leq p \leq \infty, 1 \leq q \leq \infty$. Then $\left(S_{r} g\right) * f \in \Lambda_{\alpha+2}^{p, q}$. 
Proof. $\Psi *\left(\left(S_{r} g\right) * f\right)=\left(S_{r} g\right) *(\Psi * f) \in L^{p}$, since $S_{r} g \in L^{1}$ and $\Psi * f \in L^{p}$.

And

$$
\begin{aligned}
\left\|\Phi_{n} *\left(\left(S_{r} g\right) * f\right)\right\|_{p} & =\left\|S_{r}\left(g *\left(\Phi_{n} * f\right)\right)\right\|_{p} \\
& \leq C\left\|g *\left(\Phi_{n} * f\right)\right\|_{p}
\end{aligned}
$$

But supp $\left(\Phi_{n} * f\right)^{\wedge} \subset \mathbb{A}^{n}$ which by Lemma 3.1.2 implies that

$$
\left\|g *\left(\Phi_{n} * f\right)\right\|_{p} \leq C 2^{-2 n}\left\|\Phi_{n} * f\right\|_{p} .
$$

So

$$
\sum_{n=0}^{\infty}\left(2^{n(\alpha+2)}\left\|\Phi_{n} *\left(\left(S_{r} g\right) * f\right)\right\|\right)^{q} \leq C \sum_{n=0}^{\infty}\left(2^{n \alpha}\left\|\Phi_{n} * f\right\|_{p}\right)^{q}<\infty
$$

Proposition 3.1.2. If $f \in \Lambda_{\alpha}^{p, q}(1 \leq p \leq \infty, 1 \leq q \leq \infty, \alpha \in \mathbb{R})$ and $f$ has compact support then $g * f \in \Lambda_{\alpha+2, l o c}^{p, q}$.

Proof. By Lemma 3.1.1, we need to show that if $\Omega$ is a bounded open set then there exists $h \in \Lambda_{\alpha+2}^{p, q}$ with $h=g * f$ in $\Omega$.

It is clear that for any $r>0$ there exists $C_{r}>0$ such that $g(x)=S_{r} g(x)+C_{r}$ for $|x|<r$. Since $\Omega$ is bounded and $f$ has compact support, if $r$ is large enough then $f * g=f *\left(S_{r} g+C_{r}\right)$ in $\Omega$. But $f * C_{r}$ is constant, so there exists $\psi \in C_{c}^{\infty}$ with $f * g=f * S_{r} g+\psi$ in $\Omega$. Since by Lemma $3.1 .3 f * S_{r} g \in \Lambda_{\alpha+2}^{p, q}$ and $\psi \in \Lambda_{\alpha+2}^{p, q}$ we have $f * g \in \Lambda_{\alpha+2, l o c}^{p, q}$ 
Theorem 3.1.1. Suppose $K$ is a compact subset of $\mathbb{R}^{d}, 1 \leq p \leq \infty, 1 \leq q \leq \infty$. $K$ is not $\Lambda_{\alpha, l o c}^{p, q}$-removable if and only if there exists $h \in \Lambda_{\alpha-2}^{p, q}$ such that $h \neq 0, \operatorname{supp}(h) \subseteq K$.

Proof. Suppose $K$ is not $\Lambda_{\alpha, l o c}^{p, q}$-removable. So there exists $f \in \Lambda_{\alpha, l o c}^{p, q}$ such that $f$ is harmonic in $\mathbb{R}^{d} \backslash K$ but not harmonic in $\mathbb{R}^{d}$. Let $h=\Delta f$. Then it is clear that $\operatorname{supp}(h) \subseteq K, h \neq 0$ and $h \in \Lambda_{\alpha-2}^{p, q}$.

To prove the other half of this theorem, assume that there exists $h \in \Lambda_{\alpha-2}^{p, q}$, such that $\operatorname{supp}(h) \subseteq K, h \neq 0$. Let $f=g * h$. Then $\Delta f=h$, so $f$ is harmonic in $\mathbb{R}^{d} \backslash K$, not harmonic in $\mathbb{R}^{d}$, and by Proposition 3.1.1 we have $f \in \Lambda_{\alpha, l o c}^{p, q}$.

The next theorem is an unpublished result of D. Ullrich and by courtesy of the author we are providing the proof.

Theorem 3.1.2. Suppose $K$ is a compact subset of $\mathbb{R}^{d}, \alpha<0,1 \leq p \leq \infty$ and $1 \leq q \leq \infty$. Then the following are equivalent:

(a) If $h \in \Lambda_{\alpha}^{p, q}$ and $\operatorname{supp}(h) \subseteq K$ then $h=0$;

(b) For any $\epsilon>0$ there exists $\psi_{\epsilon} \in \Lambda_{-\alpha}^{p^{\prime}, q^{\prime}}$ such that $\psi_{\epsilon}=1$ on a neighborhood of $K$ and $\left\|\psi_{\epsilon}\right\|_{p^{\prime}, q^{\prime},-\alpha}<\epsilon$

Proof. First we show that (b) implies $(a)$ : Suppose $h \in \Lambda_{\alpha}^{p, q}$ and $\operatorname{supp}(h) \subseteq K$. Consider any $\epsilon>0$ and $\psi_{\epsilon} \in \Lambda_{-\alpha}^{p^{\prime}, q^{\prime}}$ such that $\psi_{\epsilon}=1$ on a neighborhood of $K$ and $\left\|\psi_{\epsilon}\right\|_{p^{\prime}, q^{\prime},-\alpha}<\epsilon$. For any $\varphi \in C_{c}^{\infty}$, we have $\psi_{\epsilon} \varphi=\varphi$ on a neighborhood of $\operatorname{supp}(h)$, so 
from Proposition 3.1.1 follows that

$$
\begin{aligned}
|\langle h, \varphi\rangle| & =\left|\left\langle h, \psi_{\epsilon} \varphi\right\rangle\right| \\
& \leq\|h\|_{p, q, \alpha}\left\|\psi_{\epsilon} \varphi\right\|_{p^{\prime}, q^{\prime},-\alpha} \\
& \leq C\|h\|_{p, q, \alpha}\left\|\psi_{\epsilon}\right\|_{p^{\prime}, q^{\prime},-\alpha} \\
& \leq C\|h\|_{p, q, \alpha} \epsilon .
\end{aligned}
$$

Since $\epsilon$ is an arbitrary positive number, this implies that $\langle h, \phi\rangle=0$ for all $\varphi \in$ $C_{c}^{\infty}\left(\mathbb{R}^{d}\right)$, which means $h=0$.

To prove the second part of this theorem we assume that $(a)$ is right and $(b)$ is false, which will lead us to contradiction. Suppose there exists $M>0$ such that for any $\psi \in \Lambda_{-\alpha}^{p^{\prime}, q^{\prime}}$ and $\psi=1$ on a neighborhood of $K$ we have $\|\psi\|_{p^{\prime}, q^{\prime},-\alpha} \geq M$. Note that this implies $K \neq \emptyset$.

Let $\mathbb{X}=\left\{\psi \in \Lambda_{-\alpha}^{p^{\prime}, q^{\prime}}: \psi \in \mathcal{S}\right.$ and $\psi$ is constant in a neighborhood of $\left.K\right\}$. Fix $x \in K$. Define $\lambda: \mathbb{X} \rightarrow \mathbb{C}$ by $\lambda(\psi)=\psi(x)$.

It is clear that $|\lambda(\psi)| \leq \frac{1}{M}\|\psi\|_{p^{\prime}, q^{\prime},-\alpha}$ for all $\psi \in \mathbb{X}$. The Hahn-Banach theorem [4] implies that $\lambda$ extends to $\Lambda_{-\alpha, 0}^{p^{\prime}, q^{\prime}}$ with the same norm. By Theorem 1.3.5 there exists $f \in \Lambda_{\alpha}^{p, q}$ such that $\langle f, \psi\rangle=\lambda(\psi)$ for any $\psi \in \Lambda_{-\alpha, 0}^{p^{\prime}, q^{\prime}}$. Hence $\langle f, \psi\rangle=\psi(x)(x \in K, \psi \in$ $\mathbb{X})$.

Now $\lambda \neq 0$ implies that $f \neq 0$. We need to show that $\operatorname{supp}(f) \subseteq K$. This is equivalent of showing that $\langle f, \varphi\rangle=0$ for all $\varphi \in C_{c}^{\infty}, \operatorname{supp}(\varphi) \subseteq \mathbb{R}^{d} \backslash K$.

$\operatorname{But} \operatorname{supp}(\varphi) \subseteq \mathbb{R}^{d} \backslash K$ implies that $\varphi=0$ in a neighborhood of $K$, so $\varphi \in \mathbb{X}$ and $\langle f, \varphi\rangle=\varphi(x)=0$. 
Theorem 3.1.3. Suppose $K$ is a compact subset of $\mathbb{R}^{d}$ and $\alpha<2$. $K$ is $\Lambda_{\alpha, l o c^{-}}^{p, q}$ removable if and only if for any $\epsilon>0$ there exists $\psi_{\epsilon} \in \Lambda_{2-\alpha}^{p^{\prime}, q^{\prime}}, \psi_{\epsilon}=1$ on a neighborhood of $K$, and $\left\|\psi_{\epsilon}\right\|_{p^{\prime}, q^{\prime}, 2-\alpha}<\epsilon$.

Proof. This follows from theorems 3.1.1 and 3.1.2.

\section{$3.2 \beta$ and Removable Sets}

We will prove the following lemma and theorem in case when $d=2$. They can be generalized in higher dimensional Euclidean spaces easily and the proof is very similar.

We introduce following notations: $Q_{n}=\left[-2^{n-1}, 2^{n-1}\right] \times\left[-2^{n-1}, 2^{n-1}\right]$, for $n \in \mathbb{Z}$. By $\mathbb{A}_{n}^{\diamond}, n \in \mathbb{Z}$, we denote $Q_{n} \backslash Q_{n-1}$.

Proposition 3.2.1. There exists $C>0$ such that for any $h=\sum_{n=-\infty}^{\infty} h_{n}$ where $\operatorname{supp}(\hat{h}) \subset \mathbb{A}_{n}^{\diamond}$ and $\left(\sum_{n=-\infty}^{\infty}\left(2^{\alpha n}\left\|h_{n}\right\|_{p}\right)^{q}\right)^{\frac{1}{q}}<\infty(1 \leq p \leq \infty, 1 \leq q \leq \infty)$ we have

$$
\|h\|_{p, q, \alpha}^{\circ} \leq C\left(\sum_{n=-\infty}^{\infty}\left(2^{\alpha n}\left\|h_{n}\right\|_{p}\right)^{q}\right)^{\frac{1}{q}}<\infty
$$

Proof. Since $\mathbb{A}_{n}=\left\{\xi: 2^{n-1} \leq|\xi| \leq 2^{n+1}\right\}$ it is clear that

$$
\mathbb{A}_{n} \subset \mathbb{A}_{n-1}^{\diamond} \cup \mathbb{A}_{n}^{\diamond} \cup \mathbb{A}_{n+1}^{\diamond} \cup \mathbb{A}_{n+2}^{\diamond}
$$

So $h * \Phi_{n}=\left(h_{n-1}+h_{n}+h_{n+1}+h_{n+2}\right) * \Phi_{n}$. Because of this

$$
\begin{aligned}
\left\|h * \Phi_{n}\right\|_{p} & \leq\left\|\Phi_{n}\right\|_{1}\left(\left\|h_{n-1}\right\|_{p}+\left\|h_{n}\right\|_{p}+\left\|h_{n+1}\right\|_{p}+\left\|h_{n+2}\right\|_{p}\right) \\
& =\left\|\Phi_{0}\right\|_{1}\left(\left\|h_{n-1}\right\|_{p}+\left\|h_{n}\right\|_{p}+\left\|h_{n+1}\right\|_{p}+\left\|h_{n+2}\right\|_{p}\right) .
\end{aligned}
$$


All of this implies:

$$
\begin{aligned}
\|h\|_{p, q, \alpha}^{\circ} & =\left(\sum_{n=-\infty}^{\infty}\left(2^{n \alpha}\left\|\Phi_{n} *\left(h_{n-1}+h_{n}+h_{n+1}+h_{n+2}\right)\right\|_{p}\right)^{q}\right)^{\frac{1}{q}} \\
& \leq\left(\sum_{n=-\infty}^{\infty}\left(2^{n \alpha}\left\|\Phi_{0}\right\|_{1}\left(\left\|h_{n-1}\right\|_{p}+\left\|h_{n}\right\|_{p}+\left\|h_{n+1}\right\|_{p}+\left\|h_{n+2}\right\|_{p}\right)\right)^{q}\right)^{\frac{1}{q}} \\
& \leq\left\|\Phi_{0}\right\|\left(\sum_{n=-\infty}^{\infty}\left(2^{q n \alpha} 4^{q}\left(\left\|h_{n-1}\right\|_{p}^{q}+\left\|h_{n}\right\|_{p}^{q}+\left\|h_{n+1}\right\|_{p}^{q}+\left\|h_{n+2}\right\|_{p}^{q}\right)\right)\right)^{\frac{1}{q}} \\
& =4\left\|\Phi_{0}\right\|\left(\sum_{n=-\infty}^{\infty}\left(2^{q n \alpha}\left\|h_{n-1}\right\|_{p}^{q}+2^{q n \alpha}\left\|h_{n}\right\|_{p}^{q}+2^{q n \alpha}\left\|h_{n+1}\right\|_{p}^{q}+2^{q n \alpha}\left\|h_{n+2}\right\|_{p}^{q}\right)\right)^{\frac{1}{q}} \\
& \leq 4\left\|\Phi_{0}\right\|_{1} \sum_{i=-1}^{3}\left(\sum_{n=-\infty}^{\infty}\left(2^{n \alpha}\left\|h_{n+i}\right\|_{p}\right)^{q}\right)^{\frac{1}{q}} \\
& =4\left\|\Phi_{0}\right\|_{1}\left(2^{\alpha}+1+2^{-\alpha}+2^{-2 \alpha}\right)\left(\sum_{n=-\infty}^{\infty}\left(2^{\alpha n}\left\|h_{n}\right\|_{p}\right)^{q}\right)^{\frac{1}{q}} .
\end{aligned}
$$

Thus we take $C=4\left\|\Phi_{0}\right\|_{1}\left(2^{\alpha}+1+2^{-\alpha}+2^{-2 \alpha}\right)$ and we are done.

Lemma 3.2.1. Assume $Q=[-r, r] \times[-r, r], f(x)=\mathbf{1}_{[-r, r]}(x), g\left(x_{1}, x_{2}\right)=f\left(x_{1}\right) f\left(x_{2}\right)$.

Then there exists $C>0$ such that $\left\|f * \Phi_{n}\right\|_{p} \leq C 2^{-n} 2^{\frac{n}{p^{\prime}}}\left\|\Phi_{0}\right\|_{p}$ if $n>-\log _{2}(r)$ and $\left\|f * \Phi_{n}\right\|_{p} \leq C r 2^{\frac{n}{p^{\prime}}}\left\|\Phi_{0}\right\|_{p}$ if $n \leq-\log _{2}(r)$.

Proof. We know that $\hat{f}(\xi)=\frac{\sin (2 \pi r \xi)}{\pi \xi}$. We also know that $\left\|\Phi_{n}\right\|_{1}=\left\|\Phi_{0}\right\|_{1},\left\|\Phi_{n}\right\|_{\infty}=$ $2^{n}\left\|\Phi_{0}\right\|_{\infty},\left\|\Phi_{n}\right\|_{p}=2^{\frac{n}{p^{\prime}}}\left\|\Phi_{0}\right\|_{p}$ for all $n \in \mathbb{Z}$. Let us introduce a function $\varphi$ such that $\hat{\varphi}_{0}(\xi) \in C_{c}^{\infty}$ and $\hat{\varphi}_{0}(\xi)=\frac{1}{\pi \xi}$ when $\xi \in \mathbb{A}_{0}$. We define $\hat{\varphi}_{n}(\xi)=2^{-n} \hat{\varphi}_{0}\left(2^{-n} \xi\right)$ when $n \in \mathbb{N}$. Since $\left(\tau_{r} \Phi_{n}\right)^{\wedge}(\xi)=e^{-2 \pi r \xi} \hat{\Phi}_{n}(\xi)$ we have an equality

$$
f * \Phi_{n}=\left(-\tau_{r} \Phi_{n}-\tau_{-r} \Phi_{n}\right) * \varphi_{n}
$$


which implies that

$$
\begin{aligned}
\left\|f * \Phi_{n}\right\|_{p} & =\left\|\left(-\tau_{r} \Phi_{n}-\tau_{-r} \Phi_{n}\right) * \varphi_{n}\right\|_{p} \\
& \leq C\left\|\varphi_{n}\right\|_{1}\left\|\Phi_{n}\right\|_{p} \\
& =C 2^{-n}\left\|\varphi_{0}\right\|_{1}\left\|\Phi_{n}\right\|_{p} \\
& =C 2^{-n} 2^{\frac{n}{p^{\prime}}}\left\|\Phi_{0}\right\|_{p} .
\end{aligned}
$$

This finishes the first part of the lemma. The second part is easy:

$$
\left\|f * \Phi_{n}\right\|_{p} \leq\|f\|_{1}\left\|\Phi_{n}\right\|_{p} \leq C r 2^{\frac{n}{p^{\prime}}}\left\|\Phi_{0}\right\|_{1}
$$

Theorem 3.2.1. Suppose $-\frac{d}{p^{\prime}}<\alpha<\frac{1}{p}, 1<p<\infty, 1 \leq q<\infty$. Then homogeneous Besov space $\AA_{\alpha}^{p, q}$ contains the characteristic function of a d-dimensional cube $Q$ and there exists $C>0$ such that

$$
\left\|\mathbf{1}_{Q}\right\|_{p, q, \alpha}^{\circ} \leq C l(Q)^{-\alpha+\frac{d}{p}}
$$

where $l(Q)$ is the length of cube $Q$. If $q=\infty$ then we may assume $-\frac{d}{p^{\prime}} \leq \alpha \leq \frac{1}{p}$.

Proof. Recall that we are considering the case when $d=2$ and we are using following notations: $Q=[-r, r] \times[-r, r], f(x)=\mathbf{1}_{[-r, r]}(x), g\left(x_{1}, x_{2}\right)=f\left(x_{1}\right) f\left(x_{2}\right)$. If $f=$ 
$\sum_{n=-\infty}^{\infty} f_{n}\left(f_{n}=f * \Phi_{n}\right)$ then $g=\sum_{n=-\infty}^{\infty} g_{n}$ where

$$
g_{n}\left(x_{1}, x_{2}\right)=f_{n}\left(x_{1}\right) f_{n}\left(x_{2}\right)+\sum_{i=-\infty}^{n-1}\left(f_{n}\left(x_{1}\right) f_{i}\left(x_{2}\right)+f_{i}\left(x_{1}\right) f_{n}\left(x_{2}\right)\right)
$$

It is clear that

$\operatorname{supp}\left(\hat{g}_{n}\right) \subseteq\left(\operatorname{supp}\left(f_{n}\right) \times \bigcup_{i=-\infty}^{n} \operatorname{supp}\left(f_{i}\right)\right) \bigcup\left(\left(\bigcup_{i=-\infty}^{n} \operatorname{supp}\left(f_{i}\right)\right) \times \operatorname{supp}\left(f_{n}\right)\right) \subset \mathbb{A}_{n}^{\diamond}$

By proposition 3.2.1 it is enough to show that $\sum_{n=-\infty}^{\infty}\left(2^{n \alpha}\left\|g_{n}\right\|_{p}\right)^{q} \leq C l(Q)^{-\alpha q+\frac{q d}{p}}$.

By $N$ we denote the greatest integer which is smaller or equal to $-\log _{2}(r)\left(2^{N} \leq\right.$ $\left.\frac{1}{r}<2^{N+1}\right)$

$$
\begin{aligned}
\sum_{n=-\infty}^{\infty}\left(2^{n \alpha}\left\|g_{n}\right\|_{p}\right)^{q} & =\sum_{n=-\infty}^{\infty}\left(2^{n \alpha}\left\|f_{n}\left(x_{1}\right) f_{n}\left(x_{2}\right)+\sum_{i=-\infty}^{n-1}\left(f_{n}\left(x_{1}\right) f_{i}\left(x_{2}\right)+f_{i}\left(x_{1}\right) f_{n}\left(x_{2}\right)\right)\right\|_{p}\right)^{q} \\
& \leq \sum_{n=-\infty}^{\infty}\left(2^{n \alpha} \sum_{i=-\infty}^{n}\left(2\left\|f_{n}\right\|_{p}\left\|f_{i}\right\|_{p}\right)\right)^{q} \\
& =2^{q} \sum_{n=-\infty}^{N}\left(2^{n \alpha} \sum_{i=-\infty}^{n}\left(\left\|f_{n}\right\|_{p}\left\|f_{i}\right\|_{p}\right)\right)^{q}+2^{q} \sum_{n=N+1}^{\infty}\left(2^{n \alpha} \sum_{i=-\infty}^{n}\left(\left\|f_{n}\right\|_{p}\left\|f_{i}\right\|_{p}\right)\right)^{q} \\
& =2^{q} \sum_{n=-\infty}^{N}\left(2^{n \alpha}\left\|f_{n}\right\|_{p} \sum_{i=-\infty}^{n}\left\|f_{i}\right\|_{p}\right)^{q}+2^{q} \sum_{n=N+1}^{\infty}\left(2^{n \alpha}\left\|f_{n}\right\|_{p} \sum_{i=-\infty}^{n}\left\|f_{i}\right\|_{p}\right)^{q} .
\end{aligned}
$$


Let us estimate the last two terms separately:

$$
\begin{aligned}
\sum_{n=-\infty}^{N}\left(2^{n \alpha}\left\|f_{n}\right\|_{p} \sum_{i=-\infty}^{n}\left\|f_{i}\right\|_{p}\right)^{q} & \leq \sum_{n=-\infty}^{N}\left(2^{n \alpha}\left\|f_{n}\right\|_{p} \sum_{i=-\infty}^{n} 2^{\frac{i}{p^{\prime}}} r\left\|\Phi_{0}\right\|_{p}\right)^{q} \\
& \leq \sum_{n=-\infty}^{N}\left(2^{n \alpha}\left\|f_{n}\right\|_{p} C 2^{\frac{n}{p^{\prime}}} r\right)^{q} \\
& \leq C r^{q} \sum_{n=-\infty}^{N}\left(2^{n \alpha} r 2^{\frac{n}{p^{\prime}}} 2^{\frac{n}{p^{\prime}}}\right)^{q} \\
& \leq C r^{2 q} 2^{N\left(\alpha+2 \frac{1}{p^{\prime}}\right) q} \\
& =C r^{\left(2-\alpha-\frac{2}{p^{\prime}}\right) q} \\
& =C r^{-q \alpha+\frac{q 2}{p}}
\end{aligned}
$$

Note that the series are convergent when $\alpha+\frac{2}{p^{\prime}}>0$ or $\alpha>\frac{-2}{p^{\prime}}(d=2)$.

Now we estimate the second term:

$$
\begin{aligned}
\sum_{n=N+1}^{\infty}\left(2^{n \alpha}\left\|f_{n}\right\|_{p} \sum_{i=-\infty}^{n}\left\|f_{i}\right\|_{p}\right)^{q} & =\sum_{n=N+1}^{\infty}\left(2^{n \alpha}\left\|f_{n}\right\|_{p}\left(\sum_{i=-\infty}^{N}\left\|f_{i}\right\|_{p}+\sum_{i=N+1}^{n}\left\|f_{i}\right\|_{p}\right)\right)^{q} \\
& \leq \sum_{n=N+1}^{\infty}\left(2^{n \alpha}\left\|f_{n}\right\|_{p}\left(\sum_{i=-\infty}^{N} C r 2^{\frac{i}{p^{\prime}}}+\sum_{i=N+1}^{n} C 2^{-i} 2^{\frac{i}{p^{\prime}}}\right)\right)^{q} \\
& \leq C \sum_{n=N+1}^{\infty}\left(2^{n \alpha}\left\|f_{n}\right\|_{p}\left(r r^{\frac{-1}{p^{\prime}}}+2^{-n} 2^{\frac{n}{p^{\prime}}}\right)\right)^{q} \\
& \leq C \sum_{n=N+1}^{\infty}\left(2^{n \alpha} 2^{-n} 2^{\frac{n}{p^{\prime}}}\left(r r^{\frac{-1}{p^{\prime}}}+2^{-n} 2^{\frac{n}{p^{\prime}}}\right)\right)^{q} \\
& \leq C\left(^{\left(2^{N \alpha} 2^{-N} 2^{\frac{N}{p^{\prime}}}\left(r r^{\frac{-1}{p^{\prime}}}+2^{-N} 2^{\frac{N}{p^{\prime}}}\right)\right)^{q}}\right. \\
& \leq C r^{\left(-\alpha+1-\frac{1}{p^{\prime}}+1-\frac{1}{p^{\prime}}\right) q} \\
& =C r^{\left(2-\alpha-\frac{2}{p^{\prime}}\right) q} \\
& =C r^{-q \alpha+\frac{q 2}{p}} .
\end{aligned}
$$


We can see that last series converges when $\alpha-1+\frac{1}{p^{\prime}}<0$ or $\alpha<\frac{1}{p}$.

We have an analogous result for the non-homogeneous Besov spaces:

Theorem 3.2.2. If $-\frac{d}{p^{\prime}}<\alpha<\frac{1}{p}$ then for any $1 \leq p \leq \infty, 1 \leq q<\infty$, a non homogeneous Besov space $\Lambda_{\alpha}^{p, q}$ contains a characteristic function of a d-dimensional cube and there exist $C>0$ (depends on $\alpha, p, q)$ such that

$$
\left\|\mathbf{1}_{Q}\right\|_{p, q, \alpha} \leq C\left(l(Q)^{\frac{d}{p}}+l(Q)^{-\alpha+\frac{d}{p}}\right)
$$

If $q=\infty$ then we may assume $-\frac{d}{p^{\prime}} \leq \alpha \leq \frac{1}{p}$.

Proof. This proof is just a simple modification of the previous proof.

Based on this theorem we are giving a criterion of removabality property of a compact set for harmonic functions in Besov spaces when the parameter $\alpha$ is between $2-\frac{1}{p^{\prime}}$ and 2 :

Theorem 3.2.3. Suppose $K$ is a compact subset of $\mathbb{R}^{d}, 1 \leq p \leq \infty$ and $2-\frac{1}{p^{\prime}}<$ $\alpha<2$. If $m_{\beta}(K)=0$, where $\beta=(\alpha-2) p^{\prime}+d$, then $K$ is $\Lambda_{\alpha}^{p, p}$-removable.

Proof. We note that since $2-\frac{1}{p^{\prime}}<\alpha<2$ then $0<2-\alpha<\frac{1}{p^{\prime}}$ and the previous theorem implies that $\Lambda_{2-\alpha}^{p^{\prime}, p^{\prime}}$ contains characteristic functions of $d$-dimensional cubes. Let $\epsilon>0$. Since $m_{\beta}(K)=0$ there exists covering of $K$ by disjoint cubes $Q_{1}, Q_{2}, \ldots, Q_{n}$ 
such that $\sum_{i=1}^{n} l\left(Q_{i}\right)^{\beta}<\epsilon$. Then by theorem 3.2.2 we have:

$$
\begin{aligned}
\left\|\mathbf{1}_{\cup_{i=1}^{n} Q_{i}}\right\|_{p^{\prime}, q^{\prime}, 2-\alpha} & \leq\left\|\mathbf{1}_{\cup_{i=1}^{n} Q_{i}}\right\|_{p^{\prime}}+\left\|\sum_{i=1}^{n} \mathbf{1}_{Q_{i}}\right\|_{p^{\prime}, q^{\prime}, 2-\alpha}^{\circ} \\
& \leq\left(\sum_{i=1}^{n} l\left(Q_{i}\right)^{d}\right)^{\frac{1}{p^{\prime}}}+\sum_{i=1}^{n}\left\|\mathbf{1}_{Q_{i}}\right\|_{p^{\prime}, q^{\prime}, 2-\alpha}^{\circ} \\
& \leq C\left[\left(\sum_{i=1}^{n} l\left(Q_{i}\right)^{d}\right)^{\frac{1}{p^{\prime}}}+\sum_{i=1}^{n} l\left(Q_{i}\right)^{(\alpha-2) p^{\prime}+d}\right]
\end{aligned}
$$

The first term of this sum we can make less then $\frac{\epsilon}{2}$ because when $\alpha \leq 2$ then $\beta=$ $(\alpha-2) p^{\prime}+d \leq d$ and so $m_{d}(K) \leq m_{\beta}(K)$. Similarly the second term can be made less than $\frac{\epsilon}{2}$ since $m_{\beta}(K)=0$. By applying theorem 3.1.3 we finish the proof.

The previous result characterizes removable singularities in a special range of parameter $\alpha$. Next we are giving characterization of singularities in a larger range of $\alpha$ but the required condition is stronger.

Proposition 3.2.2. There exist $A>0$ and $C>0$ such that for any finite set of disjoint dyadic cubes $\Omega=\left\{Q_{1}, Q_{2}, \ldots, Q_{n}\right\}$ where $l\left(Q_{i}\right)=2^{-t_{i}},\left(t_{i} \in \mathbb{N}, i=1, \ldots, n\right)$, we can construct a function $f=\sum_{i=1}^{n} b_{i}$ with properties:

(i) $\left.f\right|_{\cup_{i=1}^{n} Q_{i}}=1$,

(ii) $\operatorname{supp}\left(b_{i}\right) \subset A Q_{i}, i=1, \ldots, n$,

(iii) $\left\|D^{\eta}\left(b_{i}\right)\right\|_{\infty} \leq C 2^{t_{i}|\eta|} t_{i}^{|\eta|}, i=1, \ldots, n$ for $|\eta| \leq 2$.

Proof. We denote by $F_{m}(m \in \mathbb{N})$ the set of cubes from $\Omega$ with side length equal to $2^{-m}$. We choose $A_{1} \in \mathbb{N}$ and let $\tilde{F}_{m}$ be the family of dyadic cubes of side length equal to $2^{-m}$ which are within $A_{1}$ cubes of a cube in $F_{m}$. This means that $Q$ is in 
$\tilde{F}_{m}$ if there exist maximum $A_{1}$ cubes of side length $2^{-m}$ such that their union is a connected subset of $\mathbb{R}^{d}$ and this group contains $Q$ and at least one cube from $F_{m}$. We choose a map $f: \tilde{F}_{m} \rightarrow F_{m}$ such that: if $Q \in \tilde{F}_{m}$ then $f(Q)$ is a cube in $F_{m}$ which is within $A_{1}$ cubes from $Q$ and in case when $Q \in F_{m}$ we make $f(Q)=Q$. For $Q_{i} \in \Omega$ we introduce the notation $\tilde{Q}_{i}=\cup_{Q \in f^{-1}\left(Q_{i}\right)} Q$. It is clear that $\tilde{Q}_{i} \cap \tilde{Q}_{k}=\emptyset$ if $Q_{i}$ and $Q_{k}$ are different cubes from $F_{m}$, and we have $\cup_{Q \in F_{m}} \tilde{Q}=\cup_{Q \in \tilde{F}_{m}} Q$.

Let us consider $p \in \mathbb{C}_{c}^{\infty}$ such that $\operatorname{supp}(p) \subseteq \overline{B(0,1)}, p(x)>0$ when $x \in B(0,1)$ and $\int_{\mathbb{R}^{d}} p(x) d x=1$. We define $p_{m}(x)=\left(\frac{2^{m}}{A_{1}}\right)^{d} p\left(\frac{2^{m} x}{A_{1}}\right)$ and $\psi_{m}=\sum_{Q \in \tilde{F}_{m}} \mathbf{1}_{Q} * p_{m}$, for any $m \in \mathbb{N}$. If $F_{m}$ is empty then we assume that $\psi_{m}=0$.

It is clear that $\psi_{m}=1$ on $Q_{i}$ if $Q_{i} \in F_{m}$. Now we show that if we choose $A_{1}$ big enough then

$$
\left\|D^{\eta}\left(\psi_{m}\right)\right\|_{\infty} \leq 2^{m|\eta|}(|\eta| \leq 2)
$$

Indeed $\psi_{m}=h * p_{m}$ where $\|h\|_{\infty}=1$ and $D^{\eta}\left(\psi_{m}\right)=h * D^{\eta}\left(p_{m}\right)$ but $D^{\eta}\left(p_{m}\right)(x)=$ $\left(\frac{2^{m}}{A_{1}}\right)^{d}\left(\frac{2^{m}}{A_{1}}\right)^{|\eta|} D^{\eta} p\left(\frac{2^{m} x}{A_{1}}\right)$. The change of variables implies that $\left\|D^{\eta}\left(p_{m}\right)\right\|_{1}=\left(\frac{2^{m}}{A_{1}}\right)^{|\eta|}\left\|D^{\eta} p\right\|_{1} \leq$ $C A_{1}^{-|\eta|} 2^{m|\eta|}$ when $|\eta| \leq 2$.

So we choose $A_{1} \in \mathbb{N}$ such that $C A_{1}^{-|\eta|} \leq 1$ when $1 \leq|\eta| \leq 2$ and we have $\left\|D^{\eta}\left(\psi_{m}\right)\right\|_{\infty} \leq\|h\|_{\infty}\left\|D^{\eta}\left(p_{m}\right)\right\|_{1} \leq 2^{m|\eta|}$ (when $\eta=0$ the inequality is trivial). We take $A=A_{1}+1$.

We will define $f$ by induction: $f_{-1}=0, f_{0}=\psi_{0}, \ldots, f_{k+1}=f_{k}+\psi_{k+1}\left(1-f_{k}\right)$. So $1-f_{k+1}=1-f_{k}-\psi_{k+1}\left(1-f_{k}\right)=\left(1-\psi_{k+1}\right)\left(1-f_{k}\right)=\left(1-\psi_{k+1}\right)\left(1-\psi_{k}\right) \ldots\left(1-\psi_{0}\right)$. 
We let $1-f=\left(1-\psi_{0}\right) \ldots\left(1-\psi_{k^{\prime}}\right)$ where $k^{\prime}$ is the biggest natural number such that there exists a cube in $\Omega$ with side length $2^{-k^{\prime}}$. We define $b_{i}=p_{t_{i}} * \mathbf{1}_{\tilde{Q}_{i}}\left(1-f_{t_{i}-1}\right), i=$ $1, \ldots, n$ where $2^{-t_{i}}=l\left(Q_{i}\right)$. It is easy to see that $f=\sum_{i=0}^{n} b_{i}$ and $\operatorname{supp}\left(b_{i}\right) \subset A Q_{i}, i=$ $1, \ldots, n$. Since $\psi_{m}(x)=1$ when $x \in \cup_{Q \in F_{m}} Q$ it follows that $\left.f\right|_{\cup_{i=1}^{n} Q_{i}}=1$ and

$$
\left|D^{\eta}\left(f_{k}\right)\right|=\left|D^{\eta}\left(1-f_{k}\right)\right|=\left|\sum_{\eta_{1}+\eta_{2}+\cdots+\eta_{k}=\eta} \prod_{j=1}^{k} D^{\eta_{j}}\left(1-\psi_{j}\right)\right|
$$

where $\eta_{1}, \eta_{2}, \ldots, \eta_{k}$ are multi-indices. So $\eta_{1} \in \mathbb{N}^{d}, \ldots, \eta_{k} \in \mathbb{N}^{d}$. It follows from equation 3.3 and an elementary combinatorial argument that

$$
\left|D^{\eta}\left(1-f_{k}\right)\right| \leq \sum_{\eta_{1}+\cdots+\eta_{k}=\eta} \prod_{j=1}^{k} 2^{k\left|\eta_{j}\right|}=2^{k|\eta|} \sum_{\eta_{1}+\cdots+\eta_{k}=\eta} 1 \leq C 2^{k|\eta|}(k+1)^{|\eta|},|\eta| \leq 2 .
$$

Hence

$$
\begin{aligned}
\left\|D^{\eta}\left(b_{i}\right)\right\|_{\infty} & =\left\|\sum_{Q \in \tilde{Q}_{i}} D^{\eta}\left(\left(1-f_{t_{i}-1}\right)\left(p_{t_{i}} * \mathbf{1}_{Q}\right)\right)\right\|_{\infty} \\
& \leq C \sum_{\omega \leq \eta}\left\|D^{\omega}\left(1-f_{t_{i}-1}\right)\right\| D^{\eta-\omega}\left(p_{t_{i}} * \mathbf{1}_{Q_{i}}\right) \|_{\infty} \\
& \leq C \sum_{\omega \leq \eta} 2^{\left(t_{i}-1\right)|\omega|} t_{i}^{|\eta|} 2^{t_{i}|\eta-\omega|} \\
& \leq C t_{i}^{|\eta|} 2^{t_{i}|\eta|},|\eta| \leq 2
\end{aligned}
$$

Based on the previous proposition, we prove 
Theorem 3.2.4. Suppose $K$ is a compact subset of $\mathbb{R}^{d}, 1 \leq p \leq \infty, 1 \leq q \leq \infty$ and $0<\alpha<2$. If there exists $\gamma \in \mathbb{R}$ such that $\gamma<\beta$ where $\beta=(\alpha-2) p^{\prime}+d,\left(\frac{1}{p}+\frac{1}{p^{\prime}}=1\right)$ and $m_{\gamma}(K)=0$ then $K$ is $\Lambda_{\alpha, l o c}^{p, q}$-removable.

Proof. We observe that $A$ is a fixed number as in the previous proposition. Let us consider arbitrary $\epsilon>0$. Since $m_{\gamma}(K)=0$ there exists a covering of $K$ by dyadic disjoint cubes $Q_{1}, Q_{2}, \ldots, Q_{n}$ such that $\sum_{i=1}^{n} 2^{-\gamma t_{i}}<\epsilon$ where $2^{-t_{i}}=l\left(Q_{i}\right), t_{i} \in \mathbb{N}, i=$ $1, \ldots, n$ (Recall that $Q$ is a $d$-dimensional cube and $l(Q)$ is its side length). By the Proposition 3.2.2 there exists $f=\sum_{i=1}^{n} b_{i}$ such that:

(i) $\left.f\right|_{K}=1$;

(ii) $\operatorname{supp}\left(b_{i}\right) \subset A Q_{i}, i=1, \ldots, n$, (iii) $\left\|D^{\eta}\left(b_{i}\right)\right\|_{\infty} \leq C 2^{|\eta| t_{i}} t_{i}^{|\eta|}, i=1, \ldots, n$ for $|\eta| \leq 2$.

We define $s_{i}=\frac{t_{i}^{2}}{2^{t_{i}\left(d / p^{\prime}-2+\alpha\right)}}(1+\sqrt{d})^{M+2}(M$ is as in definition 1.3.10) and we define $a_{i}$ by the formula

$$
s_{i} a_{i}(x)=b_{i}(x), i=1, \ldots, n .
$$

Then it is easy to see that:

(i) $\operatorname{supp}\left(a_{i}\right) \subset A Q$

$(i i)\left|D^{\eta} a_{i}(x)\right|_{\infty} \leq c 2^{t_{i}\left(\frac{d}{p^{\prime}}-(2-\alpha)+|\eta|\right)}\left(1+2^{t_{i}}\left|x-x_{Q_{i}}\right|\right)^{-M-2}$ when $|\eta| \leq 2$.

Since $\max \left(\left[d\left(\frac{1}{p}-1\right)_{+}-\alpha\right],-1\right)=-1$ when $0<\alpha<2$ it follows that $a_{i}, i=$ $1, \ldots, n$ is a $\left(2-\alpha, p^{\prime}\right)$-molecule and we can use theorem 1.3.6 to estimate $\|f\|_{p^{\prime}, p^{\prime}, 2-\alpha}^{p^{\prime}}=$ 
$\left\|\sum_{i=1}^{n} s_{i} a_{i}\right\|_{p^{\prime}, p^{\prime}, 2-\alpha}^{p^{\prime}}$ as follows:

$$
\begin{aligned}
\|f\|_{p^{\prime}, p^{\prime}, 2-\alpha}^{p^{\prime}} & \leq C \sum_{i=1}^{n}\left|s_{i}\right|^{p^{\prime}} \\
& =C \sum_{i=1}^{n}\left(t_{i}^{2 p^{\prime}} 2^{-t_{i}\left(d+(\alpha-2) p^{\prime}\right)}\right) \\
& =C \sum_{i=1}^{n}\left(t_{i}^{2 p^{\prime}} 2^{-t_{i}(\beta-\gamma)} 2^{-\gamma t_{i}}\right) \\
& \leq C \sum_{i=1}^{n} 2^{-\gamma t_{i}} \\
& \leq C \epsilon .
\end{aligned}
$$

In proving this inequality we are using the fact that without loss of generality we may assume $l\left(Q_{i}\right)=2^{-t_{i}}, i=1, \ldots, n$ so small that $t_{i}^{2 p^{\prime}} 2^{-t_{i}(\beta-\gamma)}<1$ since $\beta>\gamma$. By using theorem 3.1.3 we finish the proof.

\subsection{Summary}

The following questions need to be investigated: Suppose $K \subset \mathbb{R}^{d}$, is a compact, $0<\alpha<2, \quad, 1 \leq p \leq \infty$, and $m_{\beta}(K)=0$. In this case, is it true that $K$ is $\Lambda_{\alpha, l o c}^{p, q}$-removable? Or if $m_{\beta}(K)>0$ is it true that $K$ is not $\Lambda_{\alpha, l o c}^{p, q}$-removable?

In case when $\alpha>2+\frac{p}{d}$ we know complete answer and when $2 \leq \alpha \leq 2+\frac{p}{d}$ we only know a partial answer. The following questions still remain open: Suppose $m_{d}(K)>0$ ( $d$ is the dimension of the Euclidean space where $K$ is located). What can we say about $\Lambda_{\alpha, l o c}^{p, q}$-removability of $K$ ? How does the exact answer to this question depend on the parameters $\alpha, p$, and $q$ ? 
It seems that the Hausdorff measure is not giving exact characterization of $K$ to answer those questions and we might need more subtle measure-geometric characterization of $K$. 


\section{BIBLIOGRAPHY}

[1] L. Ahlfors, Bounded analytic functions, Duke Math. J. 14 (1947), 1-11.

[2] L. Carleson, Selected problems on exceptional sets, Van Nostrand, Princeton, N. J. (1967).

[3] G. David, Analytic capacity, Calderon-Zygmund operators, and rectifiability, Publicacions Matemátiques, Vol 43 (1999), 3-25.

[4] G. B. Folland, Real Analysis, A Wiley-Interscience Publication (1999).

[5] M. Frazier and B. Jawerth, Decomposition of Besov Spaces, Indiana University Mathematics Journal, Vol. 34 (1985), 777-799.

[6] J. Garnett, Positive length but zero analytic capacity, Proceedings AMS 21 (1970), 696-699.

[7] L. L. Helms, Introduction to potential theory, Pure and Applied Mathematics v. 22 .

[8] L. D. Ivanov, Variations of sets and functions, Nauka (1975) (in Russian).

[9] P. Jones and T. Murai, Positive analytic capacity but zero Buffon needle probability, Pacific J. Math. 133(1988), 99-114. 
[10] P. Mattila, Smooth maps, null-sets for integral geometric measure and analytic capacity, Ann.Math.123(1986), 303-309.

[11] M. Melnikov, Analytic capacity: discrete approach and curvature of measure, Math. Sbornik: Math. 186 (1995), 827-846.

[12] J. Peetre, New thoughts on Besov spaces, Duke University Mathematics Series $\mathrm{I}(1976)$.

[13] W.Rudin, Real and Complex Analysis, McGraw-Hill international editions (2004).

[14] X. Tolsa, Painleve's problem and the semiadditivity of analytic capacity, arxiv: math/0204027 v1 (2002).

[15] D. C. Ullrich, Removable sets for harmonic functions, Michigan Math. J. 38 (1991), 467-473.

[16] N. X. Uy, A removable set for Lipschitz harmonic functions, Michigan Math.J.37 (1990), 45-51.

[17] A. G. Vitushkin, Example of a set of positive length but zero analytic capacity, Dokl. Akad. Nauk SSSR 127 (1959), 246-249 (in Russian).

[18] A. G. Vitushkin, The analytic capacity of sets in problems of approximation theory, Uspekhi Mat. Nauk 22 (1967), 141-199. 


\section{VITA}

David Kighuradze

Candidate for the Degree of

Doctor of Philosophy

\section{Dissertation: REMOVABLE SETS FOR HARMONIC FUNCTIONS IN BESOV SPACES}

Major Field: Mathematics

Biographical:

Born in Tbilisi, Republic of Georgia, July 16, 1962.

Education:

Received the B.S. degree from Tbilisi State University, Tbilisi, Georgia (former soviet Union), 1984, in Mathematics

Received the M.S. degree from Tbilisi State University, Tbilisi, Georgia (former soviet Union), 1988, in Mathematics

Completed the requirements for the degree of Doctor of Philosophy with a major in Mathematics Oklahoma State University in July, 2009.

Experience:

Employed by Department of Mathematics, Tbilisi State University, as assistant Professor from July 1991 to December 1999; Employed by Department of Mathematics, Oklahoma State University, as a teaching assistant from January 2000 to July 2009. 